\title{
Synthesis of bicyclo[3.1.0]hexanes functionalized at the tip of the cyclopropane ring. Application to the synthesis of carbocyclic nucleosides
}

\author{
Maria J. Comin ${ }^{\dagger}$ Damon A. Parrish, $§$ Jeffrey R. Deschamps and Victor E. Marquez ${ }^{\star \dagger}$ \\ ${ }^{\dagger}$ Laboratory of Medicinal Chemistry, Center for Cancer Research, National Cancer Institute - Frederick, National Institutes of \\ Health, Frederick, MD 21702. §Naval Research Laboratory, Washington, DC 20375.
}

Victor Marquez [marquezv@mail.nih.gov]

\section{Part 1: Experimental procedures}

General. All chemical reagents were commercially available. Melting points were taken on a Fisher-Jones apparatus and are uncorrected. Column chromatography was performed on silica gel 60 (230-240 mesh) and analytical TLC was performed on Analtech Uniplates silica gel GF. Unless otherwise indicated, NMR spectra were determined in $\mathrm{CDCl}_{3}(99.8 \%)$ with residual $\mathrm{CHCl}_{3}$ as the reference peak (7.26 and $\left.77.0 \mathrm{ppm}\right)$ and were recorded on a Varian $400 \mathrm{MHz}$ spectrophotometer. The coupling constants are reported in Hertz, and the peak shifts are reported in the delta (ppm) scale; abbreviations s (singlet), d (doublet), dd (doublet-of-doublets), ddd (doublet-of-doublet-of-doublets), t (triplet), q (quartet) and m (multiplet). Positive-ion fastbombardment mass spectra (FABMS) were obtained on a VG 7070E mass spectrometer at an accelerating voltage of $6 \mathrm{kV}$ and a resolution of 2000. Glycerol was used as the sample matrix, and ionization was effected by a beam of xenon atoms. Elemental analyses were performed by Atlantic Microlab. Inc., Norcross, GA. Infrared spectroscopy data was obtained neat with a Jasco. FT-IR/615 spectrometer. All reaction glassware was oven dried and cooled to room temperature in a Argon atmosphere prior to use. All non-aqueous reactions were run under positive argon pressure and the reaction contents were stirred with a Teflon coated magnetic stir-bar. 


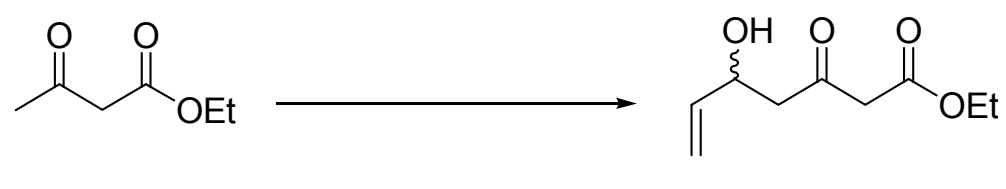

3

$( \pm)-4$

Ethyl 5-hydroxy-3-oxohept-6-enoate (( \pm -4). A stirred solution of LDA (1.9 M in THF, $49.5 \mathrm{~mL})$ in anhydrous THF (150 $\mathrm{mL})$ was treated dropwise with ethyl acetoacetate $(5 \mathrm{~mL}, 39.2 \mathrm{mmol})$ at $0{ }^{\circ} \mathrm{C}$. After $20 \mathrm{~min}$ the reaction mixture was cooled to $78^{\circ} \mathrm{C}$, and acrolein $(2.9 \mathrm{~mL}, 43.1 \mathrm{mmol})$ was added while stirring continued for $20 \mathrm{~min}$ at $-78{ }^{\circ} \mathrm{C}$. Following quenching with a saturated solution of $\mathrm{NH}_{4} \mathrm{Cl}(40 \mathrm{~mL})$, the reaction mixture was extracted with diethyl ether $(3 \mathrm{x} 150 \mathrm{~mL})$. The organic extracts were combined, dried $\left(\mathrm{MgSO}_{4}\right)$ and concentrated under vacuum. The residue was purified by flash column chromatography with hexanes:ethyl acetate (17:3) as eluant to afford $4.02 \mathrm{~g}$ (55 \% yield) of ( \pm )-4 as a colorless oil: IR (neat): 3056, 1741, 1715 $\mathrm{cm}^{-1} ;{ }^{1} \mathrm{H}$ NMR $\delta 5.86(\mathrm{ddd}, J=17.2,10.4,5.7 \mathrm{~Hz}, 1 \mathrm{H}), 5.30(\mathrm{dt}, J=17.2,1.4 \mathrm{~Hz}, 1 \mathrm{H}), 5.15(\mathrm{dt}, J=10.4,1.4 \mathrm{~Hz}, 1 \mathrm{H}), 4.60$ $(\mathrm{m}, 1 \mathrm{H}), 4.20$ (q, $J=7.2 \mathrm{~Hz}, 2 \mathrm{H}), 3.48(\mathrm{~s}, 2 \mathrm{H}), 2.78(\mathrm{mAB}, 2 \mathrm{H}), 2.74(\mathrm{~d}, J=4.1 \mathrm{~Hz}, 1 \mathrm{H}), 1.28(\mathrm{t}, J=7.2 \mathrm{~Hz}, 3 \mathrm{H}) ;{ }^{13} \mathrm{C}$ NMR: $\delta 202.7,166.8,138.7,115.3,68.5,61.5,49.9,49.2,14.1 ; \mathrm{FAB}$ MS m/z (relative intensity): $187\left(39, \mathrm{MH}^{+}\right), 169$ (69). Anal. Calcd. for $\mathrm{C}_{9} \mathrm{H}_{14} \mathrm{O}_{4}$ : C, 58.08; H, 7.58. Found: C, 57.83; H 7.58.

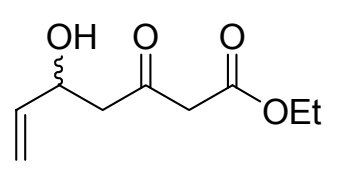

$( \pm)-4$

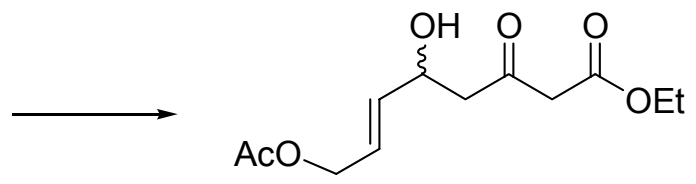

$( \pm)-5 a$

Ethyl (6E)-8-acetyloxy-5-hydroxy-3-0xooct-6-enoate $(( \pm)-5 a)$. To a stirred solution of $( \pm)-4(99 \mathrm{mg}, 0.53 \mathrm{mmol})$ in anhydrous $\mathrm{CH}_{2} \mathrm{Cl}_{2}(5 \mathrm{~mL})$ under argon, cis-1,4-diacetoxy-2-butene $(0.18 \mathrm{~mL}, 1.06 \mathrm{mmol})$ and Grubbs catalyst second generation $(22 \mathrm{mg}, 5 \% \mathrm{~mol})$ were successively added . The reaction mixture was refluxed at $45{ }^{\circ} \mathrm{C}$ overnight. The solvent was removed in vacuo and the residue was purified by flash column chromatography with hexanes:ethyl acetate (4:1) as eluant to afford $87 \mathrm{mg}$ (64 \% yield) of ( \pm )-5a as a colorless oil: IR (neat): 3503, 1731, $1711 \mathrm{~cm}^{-1} ;{ }^{1} \mathrm{H}$ NMR $\delta 5.86(\mathrm{dtd}, J=15.6,5.5,0.9$ $\mathrm{Hz}, 1 \mathrm{H}), 5.77$ (ddt, $J=15.6,5.3,1.0 \mathrm{~Hz}, 1 \mathrm{H}), 4.64(\mathrm{~m}, 1 \mathrm{H}), 4.34$ (dd, $J=5.5,1.0 \mathrm{~Hz}, 2 \mathrm{H}), 4.20$ (q, J= 7.2 Hz, $2 \mathrm{H}), 3.47$ (s, $2 \mathrm{H}), 2.79$ (m, $3 \mathrm{H}), 2.00$ (s, $3 \mathrm{H}), 1.28$ (t, $J=7.2 \mathrm{~Hz}, 3 \mathrm{H}) ;{ }^{13} \mathrm{C}$ NMR $\delta 202.6,170.7,166.8,134.5,125.2,67.4,64.0,61.6,49.9$, 49.2, 20.9, 14.1; FAB MS m/z (relative intensity): 259 (77, $\mathrm{MH}^{+}$), 241 (100), 181 (95). Anal. Calcd. for $\mathrm{C}_{12} \mathrm{H}_{18} \mathrm{O}_{6}: \mathrm{C}, 55.81 ; \mathrm{H}$, 7.02. Found: C, 55.80; H, 6.88 . 


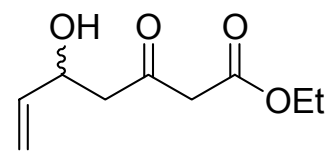

$( \pm)-4$

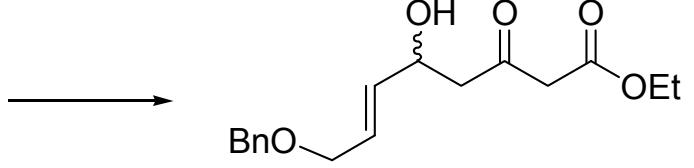

$( \pm)-5 b$

Ethyl (6E)-5-hydroxy-3-oxo-8-(phenylmethoxy)oct-6-enoate (( \pm )-5b). ( \pm )-4 (6.40 g, 34.37 mmol) was treated with cis-1,4dibencyloxy-2-butene $(18.5 \mathrm{~mL}, 69.15 \mathrm{mmol})$ following the same procedure described for the synthesis of compound $( \pm)-5 \mathbf{a}$ The reaction mixture was stirred overnight. The crude product was purified by flash column chromatography with hexanes:ethyl acetate (17:3) as eluant to afford $7.0 \mathrm{~g}$ (66 \% yield) of ( \pm )-5b as a colorless oil: IR (neat): 3440, 1739, $1712 \mathrm{~cm}^{-1}$; ${ }^{1} \mathrm{H}$ NMR $\delta 7.33(\mathrm{~m}, 5 \mathrm{H}), 5.88(\mathrm{dtd}, J=15.6,5.3,0.9 \mathrm{~Hz}, 1 \mathrm{H}), 5.77(\mathrm{ddt}, J=15.6,5.7,1.2 \mathrm{~Hz}, 1 \mathrm{H}), 4.64(\mathrm{~m}, 1 \mathrm{H}), 4.52(\mathrm{~s}, 2$ H), $4.20(\mathrm{q}, J=7.1 \mathrm{~Hz}, 2 \mathrm{H}), 4.03(\mathrm{dt}, J=5.3,1.0 \mathrm{~Hz}, 2 \mathrm{H}), 3.47(\mathrm{~s}, 2 \mathrm{H}), 2.77(\mathrm{~m}, 3 \mathrm{H}), 1.28(\mathrm{t}, J=7.1 \mathrm{~Hz}, 3 \mathrm{H}) ;{ }^{13} \mathrm{C} \mathrm{NMR} \delta$ 202.7, 166.8, 138.1, 133.1, 128.3, 127.8, 127.7, 127.0, 72.3, 69.8, 67.7, 61.5, 49.9, 49.3, 14.0; FAB MS m/z (relative intensity): 289 (14, $\left.\mathrm{MH}^{+}-\mathrm{H}_{2} \mathrm{O}\right), 199$ (12), 181 (16). Anal. Calcd. for $\mathrm{C}_{17} \mathrm{H}_{22} \mathrm{O}_{5}$ : C, 66.65; H, 7.24. Found: C, 66.65; H, 7.33.

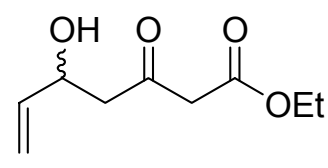

$( \pm)-4$

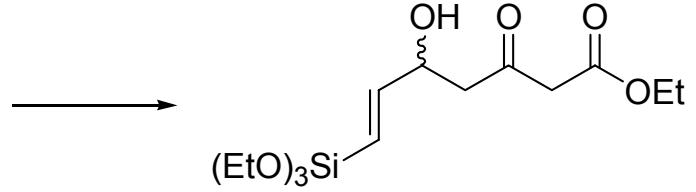

$( \pm)-5 c$

Ethyl (6E)-8,8,8-triethoxy-5-hydroxy-3-oxo-8-silaoct-6-enoate $(( \pm)-5 \mathrm{c}) .( \pm)-4(728 \mathrm{mg}, 3.91 \mathrm{mmol})$ was treated with triethoxyvinylsilane $(4.2 \mathrm{~mL}, 19.55 \mathrm{mmol})$ following the same procedure described for the synthesis of compound $( \pm)-5 \mathbf{a}$. The reaction mixture was stirred for $1 \mathrm{~h}$. The crude product was purified by flash column chromatography with hexanes:ethyl acetate (9:1) as eluant to afford $1.39 \mathrm{~g}\left(98 \%\right.$ yield) of $( \pm)-5 \mathbf{c}$ as a colorless oil: IR (neat): $3460,1743 \mathrm{~cm}^{-1} ;{ }^{1} \mathrm{H}$ NMR $\delta 6.41$ (dd, $J=18.9,4.3 \mathrm{~Hz}, 1 \mathrm{H}), 5.74(\mathrm{dd}, J=18.9,1.6 \mathrm{~Hz}, 1 \mathrm{H}), 4.63(\mathrm{~m}, 1 \mathrm{H}), 4.18(\mathrm{q}, J=7.1 \mathrm{~Hz}, 2 \mathrm{H}), 3.80(\mathrm{~m}, 6 \mathrm{H}), 3.47(\mathrm{~s}, 2 \mathrm{H})$, $2.82(\mathrm{bs}, 1 \mathrm{H}), 2.81(\mathrm{dd}, J=17.3,3.6 \mathrm{~Hz}, 1 \mathrm{H}), 2.73(\mathrm{dd}, J=17.3,8.5 \mathrm{~Hz}, 1 \mathrm{H}), 1.27$ (t, $J=7.1 \mathrm{~Hz}, 3 \mathrm{H}), 1.21(\mathrm{t}, J=7.0 \mathrm{~Hz}, 9$ $\mathrm{H}) ;{ }^{13} \mathrm{C}$ NMR $\delta 202.5,166.8,151.8,119.0,69.2,61.5,58.5,49.9,48.8,18.1,14.0 ;$ FAB MS m/z (relative intensity): 349 (11, $\left.\mathrm{MH}^{+}\right), 285$ (28). Compound ( \pm )-5c was contaminated with a ruthenium-containing salt or compound, which complicated and obscured the spectrum of the desired compound, so no accurate mass determinations were attempted for this compound. 


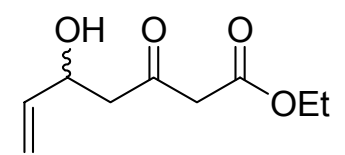

$( \pm)-4$

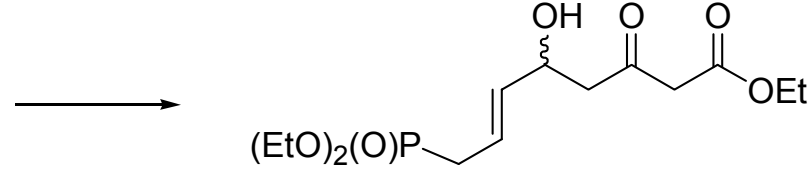

$( \pm)-5 d$

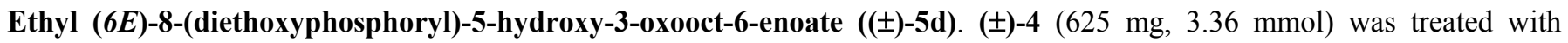
ethoxyallylphosphonate $(3.0 \mathrm{~mL}, 16.78 \mathrm{mmol})$ following the same procedure described for the synthesis of compound $( \pm)-5 \mathbf{a}$. The reaction mixture was stirred overnight. The crude product was purified by flash column chromatography with hexanes:ethyl acetate (1:4) as eluant to afford $1.14 \mathrm{~g}\left(88 \%\right.$ yield) of ( \pm )-5d as a colorless oil: IR (neat): 3357, 1744, $1712 \mathrm{~cm}^{-1}$; ${ }^{1} \mathrm{H}$ NMR $\delta 5.68(\mathrm{~m}, 2 \mathrm{H}), 4.58(\mathrm{~m}, 1 \mathrm{H}), 4.18(\mathrm{q}, J=7.1 \mathrm{~Hz}, 2 \mathrm{H}), 4.08(\mathrm{~m}, 4 \mathrm{H}), 3.47$ (s, $\left.2 \mathrm{H}\right), 2.75(\mathrm{~m} A B, 2 \mathrm{H}), 2.59-2.52(\mathrm{~m}$, $2 \mathrm{H}), 1.30(\mathrm{t}, J=7.0 \mathrm{~Hz}, 6 \mathrm{H}), 1.26(\mathrm{t}, J=7.1 \mathrm{~Hz}) ;{ }^{13} \mathrm{C}$ NMR $\delta 202.5,166.8,136.1(\mathrm{~d}, J=14.1 \mathrm{~Hz}), 120.6(\mathrm{~d}, J=11.1 \mathrm{~Hz}), 67.8$ $(\mathrm{d}, J=1.7 \mathrm{~Hz}), 62.0(\mathrm{~d}, J=6.8 \mathrm{~Hz}), 61.4,49.9,49.3(\mathrm{~d}, J=2.9 \mathrm{~Hz}), 30.0(\mathrm{~d}, J=140.0 \mathrm{~Hz}), 16.4(\mathrm{~d}, J=5.6 \mathrm{~Hz}), 14.0$; FAB MS m/z (relative intensity): $337\left(100, \mathrm{MH}^{+}\right), 319$ (36), 273 (78). Anal. Calcd. for $\mathrm{C}_{14} \mathrm{H}_{25} \mathrm{O}$ - P.0.2 $\mathrm{H}_{2} \mathrm{O}: \mathrm{C}, 49.47 ; \mathrm{H}, 7.53$. Found: C, 49.47; H, 7.63 .

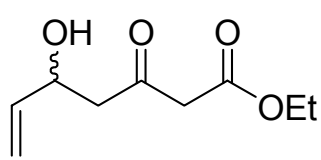

$( \pm)-4$

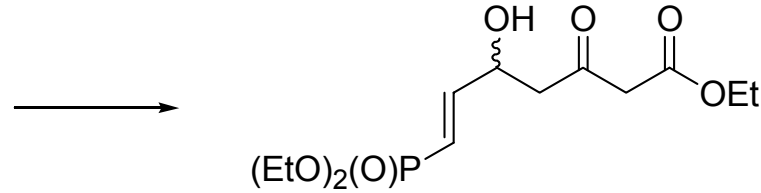

$( \pm)-5 e$

Ethyl (6E)-7-(diethoxyphosphoryl)-5-hydroxy-3-oxohept-6-enoate (( \pm )-5e). $( \pm)-4(202 \mathrm{mg}, 1.10 \mathrm{mmol})$ was treated with ethoxyvinylphosphonate $(0.9 \mathrm{~mL}, 5.8 \mathrm{mmol})$ following the same procedure described for the synthesis of compound $( \pm)-5 \mathbf{a}$. The reaction mixture was stirred for $48 \mathrm{~h}$. The crude product was purified by flash column chromatography with hexanes:ethyl acetate (1:4) as eluant to afford $166 \mathrm{mg}\left(47 \%\right.$ yield) of $( \pm)-5 \mathbf{e}$ as a colorless oil: IR (neat): $3500,3342,1713 \mathrm{~cm}^{-1} ;{ }^{1} \mathrm{H}$ NMR $\delta$ $6.72(\mathrm{ddd}, J=22.2,17.0,3.5 \mathrm{~Hz}, 1 \mathrm{H}), 6.04$ (ddd, $J=19.9,17.0,1.7 \mathrm{~Hz}, 1 \mathrm{H}), 4.75(\mathrm{~m}, 1 \mathrm{H}), 4.20$ (q, $J=7.1 \mathrm{~Hz}, 2 \mathrm{H}), 4.06$ (m, $4 \mathrm{H}), 3.48$ (s, $2 \mathrm{H}), 3.44$ (br s, $1 \mathrm{H}), 2.85$ (dd, $J=17.5,3.4 \mathrm{~Hz}, 1 \mathrm{H}), 2.75$ (dd, $J=17.5,3.4 \mathrm{~Hz}, 1 \mathrm{H}), 1.29$ (m, $9 \mathrm{H}) ;{ }^{13} \mathrm{C} \mathrm{NMR} \delta$ 202.1, 166.7, $151.9(\mathrm{~d}, J=5.6 \mathrm{~Hz}), 116.6(\mathrm{~d}, J=188.5 \mathrm{~Hz}), 67.1(\mathrm{~d}, J=21.6 \mathrm{~Hz}), 61.9(\mathrm{~d}, J=5.7 \mathrm{~Hz}), 61.7,49.7,48.3,16.3(\mathrm{~d}$, $J=6.5 \mathrm{~Hz}$ ), 14.1; FAB MS m/z (relative intensity): $323\left(100, \mathrm{MH}^{+}\right), 169$ (13). Anal Calcd. for $\mathrm{C}_{13} \mathrm{H}_{23} \mathrm{O}_{7} \mathrm{P}: \mathrm{C}, 48.45 ; \mathrm{H}, 7.19$. Found: C, 48.37; H, 7.15 . 


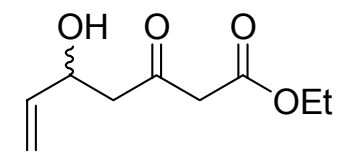

$( \pm)-4$

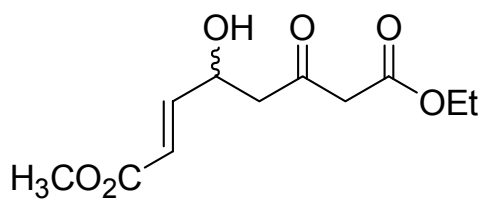

$( \pm)-5 f$

Ethyl methyl (2E)-4-hydroxy-6-oxooct-2-ene-1,8-dioate (( \pm )-5f). ( \pm )-4 (518 mg, $2.78 \mathrm{mmol})$ was treated with methylacrylate $(0.5 \mathrm{~mL}, 5.56 \mathrm{mmol})$ following the same procedure described for the synthesis of compound ( \pm )-5a. The reaction mixture was stirred overnight. The crude product was purified by flash column chromatography with hexanes:ethyl acetate (7:3) as eluant to afford $679 \mathrm{mg}$ (95\% yield) of ( \pm )-5f as a colorless oil: IR (neat): $3516,1714 \mathrm{~cm}^{-1} ;{ }^{1} \mathrm{H}$ NMR $\delta 6.89(\mathrm{dd}, J=15.6,4.2 \mathrm{~Hz}, 1 \mathrm{H})$, $6.14(\mathrm{dd}, J=15.6,1.9 \mathrm{~Hz}, 1 \mathrm{H}), 4.80(\mathrm{~m}, 1 \mathrm{H}), 4.21(\mathrm{q}, J=7.1 \mathrm{~Hz}, 2 \mathrm{H}), 3.74(\mathrm{~s}, 3 \mathrm{H}), 3.48(\mathrm{~s}, 2 \mathrm{H}), 3.03(\mathrm{~d}, J=4.1 \mathrm{~Hz}, 1 \mathrm{H})$, $2.88(\mathrm{dd}, J=17.8,3.3 \mathrm{~Hz}, 1 \mathrm{H}), 2.78(\mathrm{dd}, J=17.8,8.7 \mathrm{~Hz}, 1 \mathrm{H}), 1.29(\mathrm{t}, J=7.1 \mathrm{~Hz}, 3 \mathrm{H}) ;{ }^{13} \mathrm{C}$ NMR $\delta 202.1,166.7,166.6$, 147.6, 120.6, 66.5, 61.7, 51.7, 49.7, 48.4, 14.0; FAB MS m/z (relative intensity): 245 (66, $\mathrm{MH}^{+}$), 227 (30), 149 (76). Anal. Calcd. for $\mathrm{C}_{11} \mathrm{H}_{16} \mathrm{O}_{6}: \mathrm{C}, 54.09 ; \mathrm{H}, 6.60$. Found: $\mathrm{C}, 54.01 ; \mathrm{H}, 6.49$.

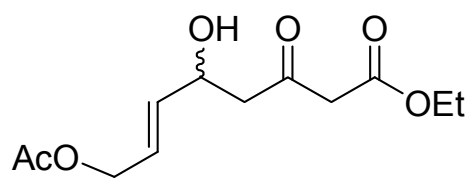

$( \pm)-5 a$

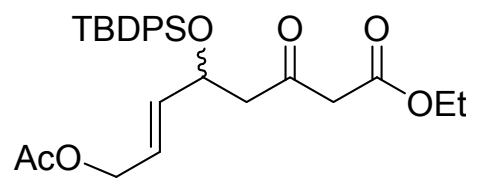

$( \pm)-6 a$

Ethyl (6E)-8-acetyloxy-5-(2,2-dimethyl-1,1-diphenyl-1-silapropoxy)-3-oxooct-6-enoate (( \pm )-6a). To a stirred solution of ( \pm )-5a (77 mg, $0.30 \mathrm{mmol})$ in anhydrous $\mathrm{CH}_{2} \mathrm{Cl}_{2}(5 \mathrm{~mL})$ under argon at $0{ }^{\circ} \mathrm{C}$, imidazole $(61 \mathrm{mg}, 0.90 \mathrm{mmol})$ and TBDPSCl $(0.12 \mathrm{~mL}, 0.45 \mathrm{mmol})$ were successively added. The reaction mixture was stirred $30 \mathrm{~min}$ at the indicated temperature and then at room temperature overnight. Brine $(5 \mathrm{~mL})$ and $\mathrm{CH}_{2} \mathrm{Cl}_{2}(10 \mathrm{~mL})$ were added and the organic phase was washed with brine $(2$ x $5 \mathrm{~mL})$, dried $\left(\mathrm{MgSO}_{4}\right)$ and concentrated under reduced pressure. The residue was purified by flash column chromatography (silica gel) employing hexanes:ethyl acetate (19:1) as eluant to afford $131 \mathrm{mg}$ (89\% yield) of ( \pm )-6a as a colorless oil: IR (neat): $1741 \mathrm{~cm}^{-1} ;{ }^{1} \mathrm{H}$ NMR $\delta$ 7.66-7.61 (m, $\left.4 \mathrm{H}\right), 7.43-7.34(\mathrm{~m}, 6 \mathrm{H}), 5.68(\mathrm{ddt}, J=15.5,6.6,1.4 \mathrm{~Hz}, 1 \mathrm{H}), 5.45(\mathrm{~m}, 1 \mathrm{H}), 4.66$ (q, $J=6.3 \mathrm{~Hz}, 1 \mathrm{H}), 4.34$ (d, $J=5.9 \mathrm{~Hz}, 2 \mathrm{H}), 4.16(\mathrm{q}, J=7.1 \mathrm{~Hz}, 2 \mathrm{H}), 3.35(\mathrm{~s}, 2 \mathrm{H}), 2.77$ (dd, $J=15.7,6.1 \mathrm{~Hz}, 1 \mathrm{H}), 2.67$ (dd, $J=15.7,6.4 \mathrm{~Hz}), 2.01(\mathrm{~s}, 3 \mathrm{H}), 1.20(\mathrm{t}, J=7.1 \mathrm{~Hz}, 3 \mathrm{H}), 1.04(\mathrm{~s}, 9 \mathrm{H}) ;{ }^{13} \mathrm{C}$ NMR $\delta 200.3,170.5,166.8,135.9,135.8,134.9$, 133.5, 133.4, 129.9, 129.7, 127.7, 127.5, 125.2, 69.9, 63.8, 61.3, 50.7, 50.3, 26.9, 20.9, 19.2, 14.1; FAB MS m/z (relative intensity): $497\left(4, \mathrm{MH}^{+}\right), 439$ (9), 241 (100). Anal. Calcd. for $\mathrm{C}_{28} \mathrm{H}_{36} \mathrm{O}_{6} \mathrm{Si}: \mathrm{C}, 67.71 ; \mathrm{H}, 7.31$. Found: C, 67.92; H, 7.40. 


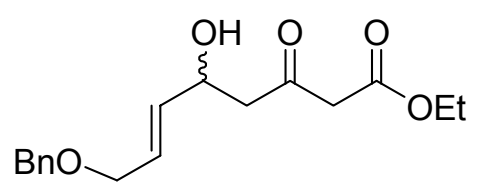

$( \pm)-5 b$

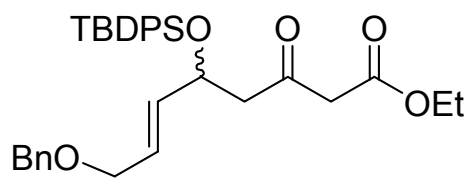

$( \pm)-6 b$

Ethyl (6E)-5-(2,2-dimethyl-1,1-diphenyl-1-silapropoxy)-3-oxo-8-(phenylmethoxy)oct-6-enoate ( \pm )-6b). Compound $( \pm)-6 b$ was obtained from compound $( \pm)-5 \mathbf{b}(7.0 \mathrm{~g} ; 22.80 \mathrm{mmol})$ following the same procedure described for the synthesis of compound ( \pm )-6a. After column chromatography purification employing hexanes:ethyl acetate (49:1) as eluant, $11.80 \mathrm{~g}(95 \%$ yield) of pure compound ( \pm )-6b was obtained as a colorless oil: IR (neat): 1742, $1717 \mathrm{~cm}^{-1} ;{ }^{1} \mathrm{H}$ NMR $\delta 7.69-7.64(\mathrm{~m}, 4 \mathrm{H})$, 7.45-7.24 (m, $11 \mathrm{H}), 5.69(\mathrm{~m}, 1 \mathrm{H}), 5.47(\mathrm{~m}, 1 \mathrm{H}), 4.69$ (q, $J=6.3 \mathrm{~Hz}, 1 \mathrm{H}), 4.33$ (s, $2 \mathrm{H}), 4.15$ (q, J= 7.2 Hz, $2 \mathrm{H}), 3.82$ (m, 2 $\mathrm{H}), 3.36(\mathrm{~s}, 2 \mathrm{H}), 2.78(\mathrm{dd}, J=15.6,6.2 \mathrm{~Hz}, 1 \mathrm{H}), 2.67(\mathrm{dd}, J=15.6,6.2 \mathrm{~Hz}, 1 \mathrm{H}), 1.24(\mathrm{t}, J=7.2 \mathrm{~Hz}, 3 \mathrm{H}), 1.05(\mathrm{~s}, 9 \mathrm{H}) ;{ }^{13} \mathrm{C}$ NMR $\delta 200.5,166.9,135.9,133.5,129.8,129.6,128.3,128.0,127.6,127.6,127.5,127.5,71.8,70.2,69.6,61.3,51.0,50.3$, 26.9, 19.3, 14.1; FAB MS m/z (relative intensity): 545 (7, $\left.\mathrm{MH}^{+}\right), 487$ (88). Anal. Calcd. for $\mathrm{C}_{33} \mathrm{H}_{40} \mathrm{O}_{5} \mathrm{Si}$ : C, 72.76; H, 7.40. Found: C, 72.94; H, 7.50.

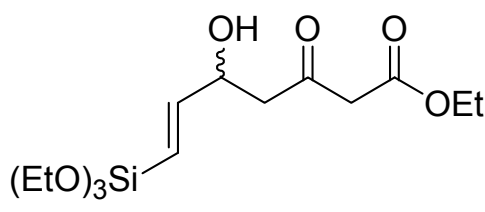

$( \pm)-5 c$

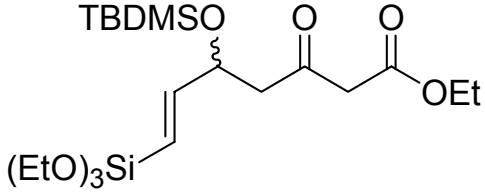

$( \pm)-6 c$

Ethyl (6E)-8,8,8-triethoxy-3-0xo-5-(1,1,2,2-tetramethyl-1-silapropoxy)-8-silaoct-6-enoate (( \pm )-6c). Compound ( \pm )-6c was obtained from compound ( \pm )-5c $(300 \mathrm{mg} ; 0.86 \mathrm{mmol})$ following the same procedure described for the synthesis of compound ( \pm )-6a. After column chromatography purification employing hexanes:ethyl acetate (25:1) as eluant, $239 \mathrm{mg}(60 \%$ yield) of pure compound ( $( \pm)-6 \mathbf{b}$ was obtained as a colorless oil: IR (neat): $1749 \mathrm{~cm}^{-1} ;{ }^{1} \mathrm{H}$ NMR $\delta 6.38(\mathrm{dd}, J=18.7,5.1 \mathrm{~Hz}, 1 \mathrm{H}), 5.66$ (dd, $J=18.7,1.4 \mathrm{~Hz}, 1 \mathrm{H}), 4.65$ (m, $1 \mathrm{H}), 4.18$ (irregular q, $2 \mathrm{H}), 3.80$ (irregular q, $6 \mathrm{H}), 3.46$ (s, $2 \mathrm{H}), 2.77$ (dd, $J=15.3,7.8$ $\mathrm{Hz}, 1 \mathrm{H}), 2.60$ (dd, $J=15.3,4.5 \mathrm{~Hz}, 1 \mathrm{H}), 1.27$ (irregular t, $3 \mathrm{H}), 1.22$ (irregular t, $9 \mathrm{H}), 0.88$ (s, $9 \mathrm{H}), 0.04$ (s, $3 \mathrm{H}), 0.03$ (s, 3 $\mathrm{H}) ;{ }^{13} \mathrm{C}$ NMR $\delta 200.9,166.9,153.3,118.7,71.2,61.3,58.5,50.9,50.4,25.8,18.2,18.1,14.1,-4.6,-5.2 ;$ FAB MS m/z (relative intensity): $463\left(4, \mathrm{MH}^{+}\right), 417$ (14), 359 (17). HRFABMS calculated for $\mathrm{C}_{21} \mathrm{H}_{43} \mathrm{O}_{7} \mathrm{Si}_{2}\left(\mathrm{MH}^{+}\right): \mathrm{m} / \mathrm{z}=463.255$; found: $\mathrm{m} / \mathrm{z}=$ 463.252. HRFABMS calculated for $\mathrm{C}_{21} \mathrm{H}_{42} \mathrm{O}_{7} \mathrm{Si}_{2} \mathrm{~K}\left(\mathrm{M}+\mathrm{K}^{+}\right): \mathrm{m} / \mathrm{z}=501.211$; found: $\mathrm{m} / \mathrm{z}=501.207$. 


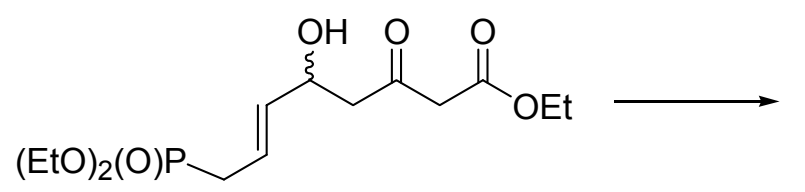

$( \pm)-5 d$

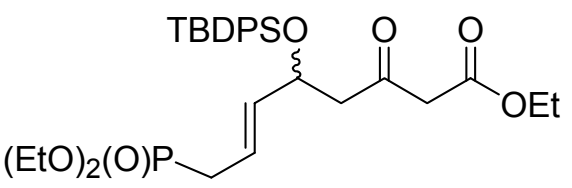

$( \pm)-6 d$

Ethyl (6E)-8-(diethoxyphosphoryl)-5-(2,2-dimethyl-1,1-diphenyl-1-silapropoxy)-3-oxooct-6-enoate (( \pm )-6d). Compound ( \pm )-6d was obtained from compound $( \pm)-5 \mathbf{d}(1.00 \mathrm{~g} ; 2.97 \mathrm{mmol})$ following the same procedure described for the synthesis of compound ( \pm )-6a. After column chromatography purification employing hexanes:ethyl acetate (1:1) as eluant, $1.40 \mathrm{~g}(80 \%$ yield) of pure compound ( \pm )-6d was obtained as a colorless oil: IR (neat): $1719 \mathrm{~cm}^{-1} ;{ }^{1} \mathrm{H}$ NMR $\delta 7.64(\mathrm{~m}, 4 \mathrm{H}), 7.39(\mathrm{~m}, 6 \mathrm{H})$, $5.59(\mathrm{~m}, 1 \mathrm{H}), 5.41(\mathrm{~m}, 1 \mathrm{H}), 4.63(\mathrm{~m}, 1 \mathrm{H}), 4.14(\mathrm{q}, J=7.1 \mathrm{~Hz}, 2 \mathrm{H}), 4.03(\mathrm{~m}, 4 \mathrm{H}), 3.33(\mathrm{~s}, 2 \mathrm{H}), 2.70(\mathrm{dd}, J=15.6,5.9 \mathrm{~Hz}, 1$ H), $2.63(\mathrm{dd}, J=15.6,6.4 \mathrm{~Hz}, 1 \mathrm{H}), 2.45-2.34(\mathrm{~m}, 2 \mathrm{H}$ ) $), 1.26(\mathrm{~m}, 9 \mathrm{H}), 1.04(\mathrm{~s}, 9 \mathrm{H}) ;{ }^{13} \mathrm{C}$ NMR $\delta 200.4,166.9,136.1(\mathrm{~d}, J=$ 14.6 Hz), 135.9, 135.8, 133.5, 129.9, 129.7, 127.7, 127.5, 120.8 (d, $J=10.7 \mathrm{~Hz}), 70.2(\mathrm{C}-5), 61.8(\mathrm{~m}), 61.2,50.8(\mathrm{~d}, J=2.8$ Hz), 50.3, 29.9 (d, $J=138.8 \mathrm{~Hz}), 26.9,19.2,16.4(\mathrm{~m}), 14.1$; FAB MS m/z (relative intensity): 575 (21, $\left.\mathrm{MH}^{+}\right), 471$ (48). Anal. Calcd. for $\mathrm{C}_{30} \mathrm{H}_{43} \mathrm{O}_{7} \mathrm{PSi} 0.3 \mathrm{H}_{2} \mathrm{O}: \mathrm{C}, 62.11 ; \mathrm{H}, 7.58$. Found: C, 61.96; H, 7.58.

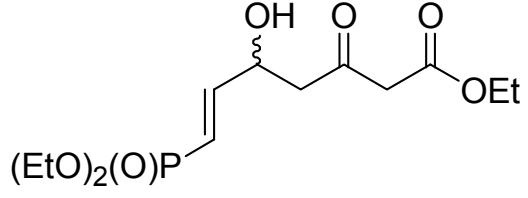

( \pm -5e

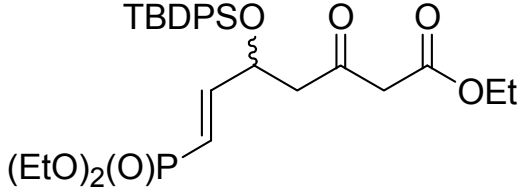

(土)-6e

Ethyl (6E)-7-(diethoxyphosphoryl)-5-(2,2-dimethyl-1,1-diphenyl-1-silapropoxy)-3-oxohept-6-enoate (( \pm )-6e). Compound ( \pm )-6e was obtained from compound $( \pm)-5 e(75 \mathrm{mg} ; 0.23 \mathrm{mmol})$ following the same procedure described for the synthesis of compound ( \pm )-6a. After column chromatography purification employing hexanes:ethyl acetate (3:2) as eluant, $103 \mathrm{mg}$ (79\% yield) of pure compound $\left( \pm\right.$ )-6e was obtained as a colorless oil: IR (neat): 1745, 1718, $1024 \mathrm{~cm}^{-1} ;{ }^{1} \mathrm{H}$ NMR $\delta$ 7.67-7.59 (m, 4 H), 7.44-7.34 (m, $6 \mathrm{H}), 6.72(\mathrm{ddd}, J=21.9,17.0,4.9 \mathrm{~Hz}, 1 \mathrm{H}), 6.84(\mathrm{~m}, 1 \mathrm{H}), 4.78(\mathrm{~m}, 1 \mathrm{H}), 4.14$ (q, $J=7.1 \mathrm{~Hz}, 2 \mathrm{H}), 3.96(\mathrm{~m}$, $4 \mathrm{H}), 3.25$ (s, $2 \mathrm{H}), 2.72(\mathrm{mAB}, 2 \mathrm{H}), 1.29-1.22(\mathrm{~m}, 9 \mathrm{H}), 1.06$ (s, $9 \mathrm{H}) ;{ }^{13} \mathrm{C}$ NMR $\delta$ 199.4, 166.6, 152.2 (d, J = 5.3 Hz), 135.8, 135.7, 133.0, 132.8, 130.1, 129.9, 127.8, 127.7, $119.6(\mathrm{~d}, J=186.8 \mathrm{~Hz}), 69.5(\mathrm{~d}, J=22.7 \mathrm{~Hz}), 69.4(\mathrm{~d}, J=22.6 \mathrm{~Hz}), 61.7(\mathrm{~m})$, 61.4, 49.9, 49.8, 26.9, 19.3, 16.3 (d, J = 6.4 Hz), 14.0; FAB MS m/z (relative intensity): 561 (84, $\left.\mathrm{MH}^{+}\right), 457$ (49). Anal. Calcd. for $\mathrm{C}_{29} \mathrm{H}_{41} \mathrm{O}_{7} \mathrm{PSi}$ : C, 62.12; H, 7.37. Found: C, 62.36; H; 7.17. 


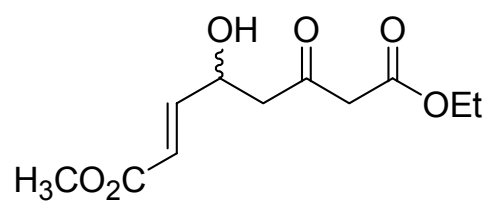

$( \pm)-5 f$

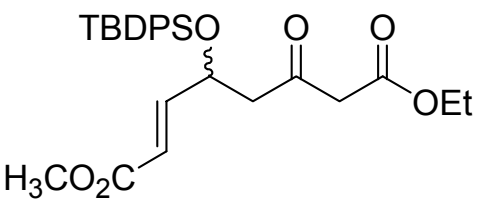

$( \pm)-6 f$

Ethyl methyl (2E)-4-(2,2-dimethyl-1,1-diphenyl-1-silapropoxy)-6-oxooct-2-ene-1,8-dioate (( \pm )-6f). Compound ( \pm )-6f was obtained from compound ( \pm )-5f (550 $\mathrm{mg} ; 2.25 \mathrm{mmol})$ following the same procedure described for the synthesis of compound (士)-6a. After column chromatography purification employing hexanes:ethyl acetate (19:1) as eluant, $923 \mathrm{mg}$ (85\% yield) of pure compound ( \pm )-6f was obtained as a colorless oil: IR (neat): 2860, $1722 \mathrm{~cm}^{-1} ;{ }^{1} \mathrm{H}$ NMR $\delta$ 7.69-7.57 (m, $\left.4 \mathrm{H}\right), 7.45-7.30$ (m, $6 \mathrm{H}), 6.87(\mathrm{dd}, J=15.6,5.3 \mathrm{~Hz}, 1 \mathrm{H}), 5.93(\mathrm{dd}, J=15.6,1.5 \mathrm{~Hz}, 1 \mathrm{H}), 4.81(\mathrm{~m}, 1 \mathrm{H}), 4.13(\mathrm{q}, J=7.2 \mathrm{~Hz}, 2 \mathrm{H}), 3.70(\mathrm{~s}, 3 \mathrm{H})$, $3.25(m A B, 2 \mathrm{H}), 2.70(m A B, 2 \mathrm{H}), 1.23(\mathrm{t}, J=7.2 \mathrm{~Hz}, 3 \mathrm{H}), 1.07$ (s, $9 \mathrm{H}) ;{ }^{13} \mathrm{C}$ NMR $\delta$ 199.5, 166.5, 166.5, 148.3, 135.8, 135.7, 133.1, 132.7, 130.0, 129.9, 127.8, 127.7, 120.4, 68.5, 61.3, 51.5, 50.0, 49.9, 26.9, 19.2, 14.0; FAB MS m/z (relative intensity): $483\left(3, \mathrm{MH}^{+}\right), 379$ (15). Anal. Calcd. for $\mathrm{C}_{27} \mathrm{H}_{34} \mathrm{O}_{6} \mathrm{Si}: \mathrm{C}, 67.19 ; \mathrm{H}, 7.10$. Found: C, 67.42; H, 7.23.

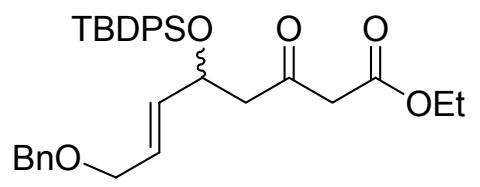

$( \pm)-6 b$

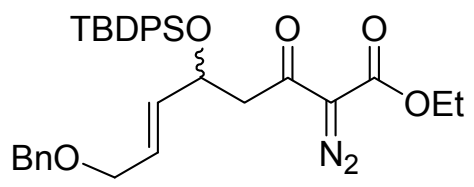

$( \pm)-7 \mathbf{b}$

Ethyl (6E)-5-(2,2-dimethyl-1,1-diphenyl-1-silapropoxy)-2-diazo-3-oxo-8-(phenylmethoxy)oct-6-enoate (( \pm )-7b). A stirred solution of $( \pm)-6 \mathbf{b}(12.4 \mathrm{~g}, 22.8 \mathrm{mmol})$ and tosyl azide $(5.0 \mathrm{~g}, 25.00 \mathrm{mmol})$ in $\mathrm{CH}_{3} \mathrm{CN}(100 \mathrm{~mL})$ was treated with triethylamine $(6.4 \mathrm{~mL}, 42.88 \mathrm{mmol})$ at $0{ }^{\circ} \mathrm{C}$. After $2 \mathrm{~h}$ of stirring at $0{ }^{\circ} \mathrm{C}$, the reaction mixture was allowed to reach room temperature and stirring was continued for $2 \mathrm{~h}$. Diethyl ether $(200 \mathrm{~mL})$ and $2 \mathrm{~N}$ aqueous $\mathrm{NaOH}(40 \mathrm{~mL})$ were added. After $10 \mathrm{~min}$, the organic layer was separated, dried $\left(\mathrm{MgSO}_{4}\right)$ and concentrated under vacuum. The residue was purified by flash column chromatography with hexanes:ethyl acetate (49:1) as eluant to afford $12.9 \mathrm{~g}$ (99 \% yield) of diazo compound ( \pm )-7b as a colorless oil: IR (neat): 2137, 1716, $1654 \mathrm{~cm}^{-1} ;{ }^{1} \mathrm{H}$ NMR $\delta$ 7.69-7.61 (m, $\left.4 \mathrm{H}\right), 7.38-7.20(\mathrm{~m}, 11 \mathrm{H}), 5.72(\mathrm{ddt}, J=15.5,7.0,1.4 \mathrm{~Hz}, 1 \mathrm{H}), 5.44(\mathrm{dtd}, J=$ 15.5, 5.7, $0.8 \mathrm{~Hz}, 1 \mathrm{H}), 4.76(\mathrm{q}, J=6.7 \mathrm{~Hz}, 1 \mathrm{H}), 4.29(\mathrm{~s}, 2 \mathrm{H}), 4.20$ (m, $2 \mathrm{H}), 3.80$ (dd, $J=5.7,1.4 \mathrm{~Hz}, 2 \mathrm{H}), 3.21$ (dd, $J=15.1$, $7.4 \mathrm{~Hz}, 1 \mathrm{H}), 2.95(\mathrm{dd}, J=15.1,5.5 \mathrm{~Hz}), 1.27$ (t, $J=7.1 \mathrm{~Hz}, 3 \mathrm{H}), 1.00(\mathrm{~s}, 9 \mathrm{H}) ;{ }^{13} \mathrm{C}$ NMR $\delta 189.9,161.1,138.3,136.0,135.9$, $134.4,133.9,133.7,129.6,129.5,128.3,127.6,127.5,127.4,127.3,71.7,70.7,69.8,61.3,48.0,26.9,19.3,14.3 ;$ FAB MS m/z (relative intensity): $571\left(1, \mathrm{MH}^{+}\right), 513$ (6). Anal. Calcd. for $\mathrm{C}_{33} \mathrm{H}_{38} \mathrm{~N}_{2} \mathrm{O}_{5} \mathrm{Si}: \mathrm{C}, 69.74 ; \mathrm{H}, 6.71 ; \mathrm{N}, 4.91$. Found: C, 69.94; H 6.52; N, 4.89 . 


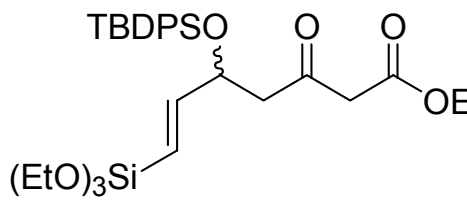

$( \pm)-6 c$

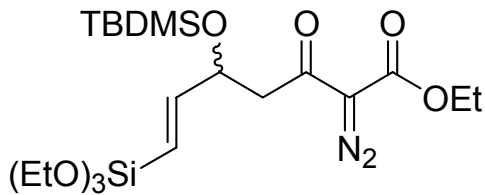

$( \pm)-7 c$

Ethyl (6E)-2-diazo-8,8,8-triethoxy-3-oxo-5-(1,1,2,2-tetramethyl-1-silapropoxy)-8-silaoct-6-enoate (( \pm )-7c). Compound $( \pm)-$ 6c $(239 \mathrm{mg}, 0.52 \mathrm{mmol})$ was treated as described for compound ( \pm )-6b to afford, after column chromatography purification (hexanes:ethyl acetate, 25:1), $255 \mathrm{mg}$ (99\% yield) of pure compound ( \pm )-7c as a colorless oil: IR (neat): $2131,1716,1656 \mathrm{~cm}^{-}$

1, ${ }^{1} \mathrm{H}$ NMR $\delta 6.44(\mathrm{dd}, J=18.9,5.1 \mathrm{~Hz}, 1 \mathrm{H}), 5.68(\mathrm{dd}, J=18.9,1.6 \mathrm{~Hz}, 1 \mathrm{H}), 4.71(\mathrm{~m}, 1 \mathrm{H}), 4.29(\mathrm{q}, J=7.0 \mathrm{~Hz}, 2 \mathrm{H}), 3.80$ (q, $J=6.8 \mathrm{~Hz}, 6 \mathrm{H}), 3.24(\mathrm{dd}, J=15.0,8.2 \mathrm{~Hz}, 1 \mathrm{H}), 2.87(\mathrm{dd}, J=15.0,4.7 \mathrm{~Hz}, 1 \mathrm{H}), 1.32(\mathrm{t}, J=7.0 \mathrm{~Hz}, 3 \mathrm{H}), 1.22(\mathrm{t}, J=7.0 \mathrm{~Hz}$, 9 H), 0.86 (s, $9 \mathrm{H}), 0.02$ (s, $3 \mathrm{H}), 0.01$ (s, $3 \mathrm{H}),{ }^{13} \mathrm{C}$ NMR $\delta$ 190.2, 161.1, 153.9, 118.2, 71.6, 61.4, 58.4, 47.5, 25.7, 18.2, 18.0, 14.0, -4.6, -5.2; FAB MS m/z (relative intensity): 489 (6, $\left.\mathrm{MH}^{+}\right), 357$ (29). HRFABMS calculated for $\mathrm{C}_{21} \mathrm{H}_{41} \mathrm{~N}_{2} \mathrm{O}_{7} \mathrm{Si}_{2}\left(\mathrm{MH}^{+}\right)$: $\mathrm{m} / \mathrm{z}=489.245$; found: $\mathrm{m} / \mathrm{z}=489.244$. HRFABMS calculated for $\mathrm{C}_{21} \mathrm{H}_{40} \mathrm{~N}_{2} \mathrm{O}_{7} \mathrm{Si}_{2} \mathrm{~K}\left(\mathrm{M}+\mathrm{K}^{+}\right): \mathrm{m} / \mathrm{z}=527.201$; found: $\mathrm{m} / \mathrm{z}=$ 527.202 .

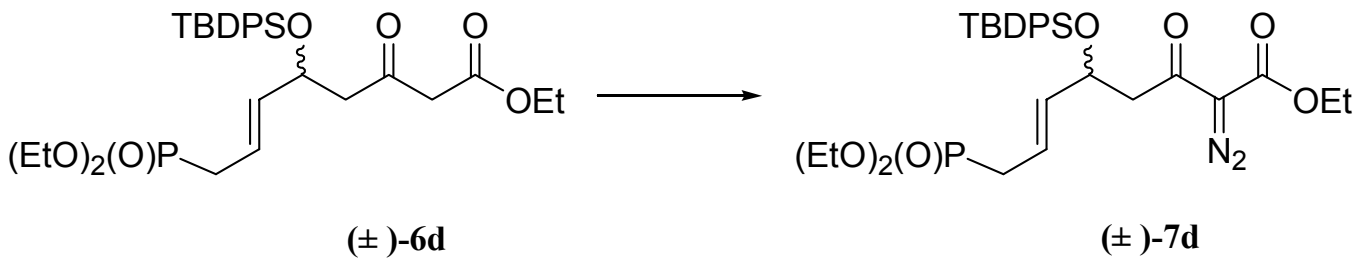

Ethyl (6E)-8-(diethoxyphosphoryl)-5-(2,2-dimethyl-1,1-diphenyl-1-silapropoxy)-2-diazo-3-oxooct-6-enoate $(( \pm)-7 d)$.

Compound ( \pm )-6d (715 mg, $1.24 \mathrm{mmol})$ was treated as described for compound ( \pm )-6b to afford, after column chromatography purification (hexanes:ethyl acetate, 7:3), $740 \mathrm{mg}$ (100\% yield) of pure compound ( \pm )-7d as a colorless oil: IR (neat): 2135, 1716, $1654 \mathrm{~cm}^{-1} ;{ }^{1} \mathrm{H}$ NMR $\delta 7.64(\mathrm{~m}, 4 \mathrm{H}), 7.40$ (m, $\left.6 \mathrm{H}\right), 5.64(\mathrm{ddd}, J=15.4,6.7,4.9 \mathrm{~Hz}, 1 \mathrm{H}), 5.39(\mathrm{~m}, 1 \mathrm{H}), 4.74(\mathrm{~m}, 1 \mathrm{H})$, $4.24(\mathrm{~m}, 2 \mathrm{H}), 4.02(\mathrm{~m}, 4 \mathrm{H}), 3.17$ (dd, $J=15.3,7.2 \mathrm{~Hz}, 1 \mathrm{H}), 2.95(\mathrm{dd}, J=15.3,5.9 \mathrm{~Hz}, 1 \mathrm{H}), 2.40(\mathrm{~m}, 2 \mathrm{H}), 1.27(\mathrm{t}, J=7.2 \mathrm{~Hz}$ $3 \mathrm{H}), 1.26$ (m, $6 \mathrm{H}), 1.02$ (s, $9 \mathrm{H}) ;{ }^{13} \mathrm{C}$ NMR $\delta$ 189.7, 161.0, 136.8 (d, $\left.J=14.3 \mathrm{~Hz}\right), 136.0,135.9,133.8,133.7,129.6,129.5$, 127.4, 127.3, 120.3 (d, $J=11.0 \mathrm{~Hz}), 70.6,61.7$ (m), 61.3, 47.8 (d, $J=2.8 \mathrm{~Hz}), 30.0$ (d, $J=138.6 \mathrm{~Hz}), 26.9,19.2,16.4$ (m), 14.3; FAB MS m/z (relative intensity): 601 (37, $\left.\mathrm{MH}^{+}\right), 169$ (24). Anal. Calcd. for $\mathrm{C}_{30} \mathrm{H}_{41} \mathrm{~N}_{2} \mathrm{O}_{7} \mathrm{PSi}$ : C, 59.88; H, 6.88; $\mathrm{N}, 4.66$. Found: C, 60.06; H, 6.92; N, 4.43. 


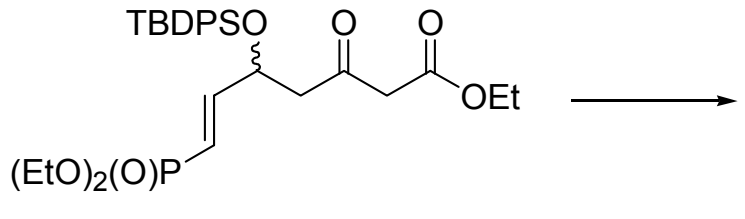

$( \pm)-6 e$

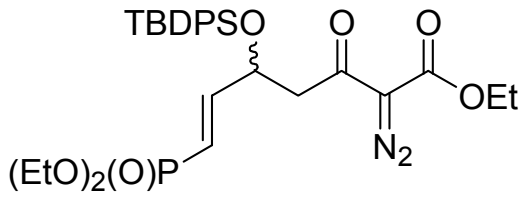

$( \pm)-7 e$

\section{Ethyl (6E)-7-(diethoxyphosphoryl)-5-(2,2-dimethyl-1,1-diphenyl-1-silapropoxy)-2-diazo-3-oxohept-6-enoate $(( \pm)-7 \mathrm{e})$.}

Compound ( \pm )-6e (333 mg, $0.59 \mathrm{mmol})$ was treated as described for compound $( \pm)$-6b to afford $343 \mathrm{mg}(99 \%$ yield) of pure compound (土)-7e as a colorless oil: IR (neat): 2136, 1716, $1654 \mathrm{~cm}^{-1} ;{ }^{1} \mathrm{H}$ NMR $\delta$ 7.65-7.60 (m, $\left.4 \mathrm{H}\right)$, 7.44-7.34 (m, $\left.6 \mathrm{H}\right), 6.79$ (ddd, $J=21.9,17.0,4.8 \mathrm{~Hz}, 1 \mathrm{H}), 5.87$ (ddd, $J=20.6,17.0,1.5 \mathrm{~Hz}, 1 \mathrm{H}), 4.91(\mathrm{~m}, 1 \mathrm{H}), 4.24(\mathrm{~m}, 2 \mathrm{H}), 3.96(\mathrm{~m}, 4 \mathrm{H}), 3.12$ (dd, $J=16.2,6.5 \mathrm{~Hz}, 1 \mathrm{H}), 3.01(\mathrm{dd}, J=16.2,6.3 \mathrm{~Hz}), 1.30(\mathrm{t}, J=7.1 \mathrm{~Hz}, 3 \mathrm{H}), 1.27(\mathrm{~m}, 3 \mathrm{H}), 1.05(\mathrm{~s}, 9 \mathrm{H}) ;{ }^{13} \mathrm{C}$ NMR $\delta 188.9$, $160.9,153.1(\mathrm{~d}, J=5.4 \mathrm{~Hz}), 135.9,135.8,133.2,133.1,129.8,129.7,127.6,116.5(\mathrm{~d}, J=186.1 \mathrm{~Hz}), 69.9(\mathrm{~d}, J=22.7 \mathrm{~Hz}), 61.6$ (d, $J=5.3 \mathrm{~Hz}), 61.4,47.2,26.9,19.2,16.3(\mathrm{~d}, J=6.5 \mathrm{~Hz}), 14.3$; FAB MS m/z (relative intensity): 587 (62, $\left.\mathrm{MH}^{+}\right), 529(11)$. Anal. Calcd. for $\mathrm{C}_{29} \mathrm{H}_{39} \mathrm{~N}_{2} \mathrm{O}_{7}$ PSi C, 59.37; H, 6.70; N, 4.77. Found: C, 59.31; H, 6.74; N, 4.70.

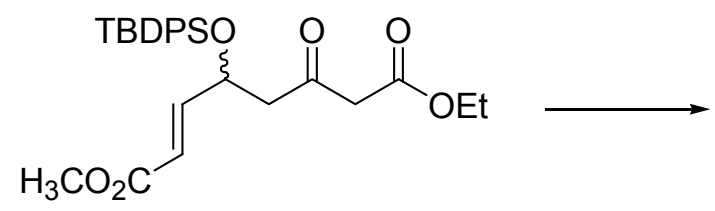

$( \pm)-6 f$

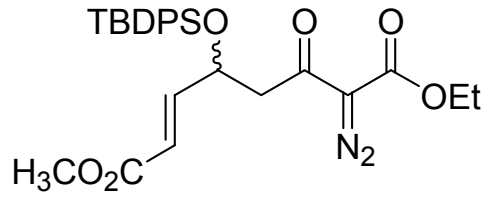

$( \pm)-7 \mathbf{f}$

Ethyl methyl (2E)-4-(2,2-dimethyl-1,1-diphenyl-1-silapropoxy)-7-diazo-6-oxooct-2-ene-1,8-dioate (( \pm )-7f). Compound ( \pm )6f $(518 \mathrm{mg}, 1.07 \mathrm{mmol})$ was treated as described for compound $( \pm)-6 \mathbf{b}$ to afford, after column chromatography purification (hexanes:ethyl acetate, 9:1), $517 \mathrm{mg}$ (95\% yield) of pure compound ( \pm )-7f as a colorless oil: IR (neat): 2136, 1716, $1654 \mathrm{~cm}^{-1}$; ${ }^{1} \mathrm{H}$ NMR $\delta$ 7.62-7.56 (m, 4 H), 7.36-7.22 (m, 6 H), $6.93(\mathrm{dd}, J=15.6,5.5 \mathrm{~Hz}, 1 \mathrm{H}), 5.94(\mathrm{dd}, J=15.6,1.4 \mathrm{~Hz}, 1 \mathrm{H}), 4.92(\mathrm{~m}, 1$ H), $4.24(\mathrm{q}, J=7.1 \mathrm{~Hz}, 2 \mathrm{H}), 3.67$ (s, $3 \mathrm{H}), 3.12(\mathrm{dd}, J=16.2,6.4 \mathrm{~Hz}, 1 \mathrm{H}), 3.02(\mathrm{dd}, J=16.2,6.4 \mathrm{~Hz}), 1.30(\mathrm{t}, J=7.1 \mathrm{~Hz}, 3$ H), 1.03 (s, $9 \mathrm{H}) ;{ }^{13} \mathrm{C}$ NMR $\delta 188.9,166.7,160.9,149.1,135.8,133.3,132.9,129.8,129.7,127.5,127.5,120.2,69.0,61.4$, 51.4, 47.3, 26.9, 19.2, 14.3; FAB MS m-/z (relative intensity): 509 (11, $\left.\mathrm{MH}^{+}\right), 451$ (21). Anal. Calcd. for $\mathrm{C}_{27} \mathrm{H}_{32} \mathrm{~N}_{2} \mathrm{O}_{6} \mathrm{Si}$ : C, 63.76; H, 6.34; N, 5.51. Found: C, 63.88; H, 6.40; N, 5.45. 


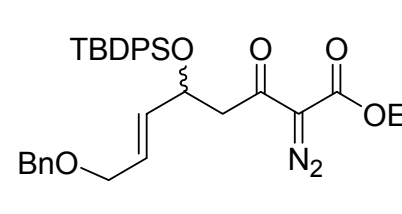

$( \pm)-7 \mathbf{b}$

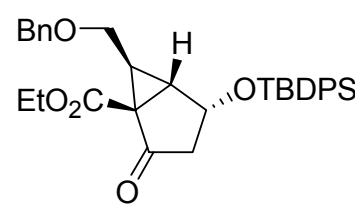

$( \pm)-8 b$

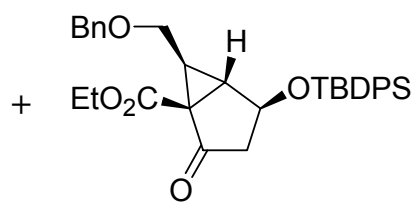

$( \pm)-9 b$

\section{Ethyl 4-(2,2-dimethyl-1,1-diphenyl-1-silapropoxy)-2-oxo-6-[(phenylmethoxy)methyl]bicyclo[3.1.0]hexanecarboxylate}

$(( \pm)-8 \mathbf{b}$ and $( \pm)-9 \mathbf{b})$. A suspension of $( \pm)-7 \mathbf{b}(13.0 \mathrm{~g}, 22.80 \mathrm{mmol})$ and anhydrous copper sulfate $(4.3 \mathrm{~g}, 25.80 \mathrm{mmol})$ in cyclohexane $(200 \mathrm{~mL})$ was stirred at reflux $\left(\mathrm{T}_{\text {bath }}=100^{\circ} \mathrm{C}\right)$ for $41 \mathrm{~h}$. After cooling to room temperature, the reaction mixture was filtered through a pad of Celite ${ }^{\circledR}$, which was then washed with diethyl ether. The solution was concentrated under vacuum and the residue was purified by column chromatography (silica gel) with hexanes:ethyl acetate (19:1) as eluant to afford $6.3 \mathrm{~g}$ of compound ( \pm )-8b and $2.9 \mathrm{~g}$ of the minor C-4 epimer ( \pm )-9b (75 \% overall yield) as colorless oils. Compound ( \pm )-8b: IR (neat): 1743, $1718 \mathrm{~cm}^{-1} ;{ }^{1} \mathrm{H}$ NMR $\delta$ 7.70-7.60 (m, $\left.4 \mathrm{H}\right)$, 7.47-7.29 (m, $\left.11 \mathrm{H}\right), 4.65(\mathrm{ddd}, J=8.4,7.4,5.3 \mathrm{~Hz}, 1 \mathrm{H}), 4.51(\mathrm{~m} A B$, $2 \mathrm{H}), 4.12(\mathrm{~m}, 2 \mathrm{H}), 3.73(\mathrm{dd}, J=10.6,5.6 \mathrm{~Hz}, 1 \mathrm{H}), 3.60(\mathrm{dd}, J=10.6,7.0 \mathrm{~Hz}, 1 \mathrm{H}), 2.55(\mathrm{t}, J=5.3 \mathrm{~Hz}, 1 \mathrm{H}), 2.43(\mathrm{ddd}, J=$ 6.9, 5.6, $5.3 \mathrm{~Hz}, 1 \mathrm{H}), 2.35$ (dd, $J=18.4,8.4 \mathrm{~Hz}, 1 \mathrm{H}), 2.25(\mathrm{dd}, J=18.4,7.4 \mathrm{~Hz}, 1 \mathrm{H}), 1.20$ (t, $J=7.1 \mathrm{~Hz}, 3 \mathrm{H}), 1.06(\mathrm{~s}, 9 \mathrm{H})$; ${ }^{13} \mathrm{C}$ NMR $\delta$ 202.2, 165.5, 137.9, 135.6, 135.5, 133.4, 133.2, 130.0, 128.4, 127.9, 127.8, 127.7, 127.5, 72.8, 66.9, 66.4, 61.5, 45.9, 42.6, 37.5, 30.6, 26.8, 19.1, 14.1; FAB MS m/z (relative intensity): 543 (6, $\left.\mathrm{MH}^{+}\right), 435$ (4). Anal. Calcd. for $\mathrm{C}_{33} \mathrm{H}_{38} \mathrm{O}_{5}$ Si.0.1 $1 \mathrm{H}_{2} \mathrm{O}: \mathrm{C}, 72.87$; H, 7.07. Found: C, 72.57; H, 7.14. Compound ( \pm )-9b: IR (neat): $1739 \mathrm{~cm}^{-1} ;{ }^{1} \mathrm{H}$ NMR $\delta$ 7.69$7.61(\mathrm{~m}, 4 \mathrm{H}), 7.46-7.22(\mathrm{~m}, 11 \mathrm{H}), 4.40(\mathrm{~d}, J=4.9 \mathrm{~Hz}, 1 \mathrm{H}), 4.37(\mathrm{~s}, 2 \mathrm{H}), 4.22(\mathrm{~m}, 2 \mathrm{H}), 3.57(\mathrm{mAB}, 2 \mathrm{H}), 2.60(\mathrm{~d}, J=5.2$ $\mathrm{Hz}), 2.30(\mathrm{dd}, J=18.8,5.0 \mathrm{~Hz}, 1 \mathrm{H}), 2.19(\mathrm{~d}, J=18.8 \mathrm{~Hz}, 1 \mathrm{H}), 1.65$ (irregular q, $1 \mathrm{H}), 1.28(\mathrm{t}, J=7.1 \mathrm{~Hz}, 3 \mathrm{H}), 1.06(\mathrm{~s}, 9 \mathrm{H})$;

${ }^{13} \mathrm{C}$ NMR $\delta 204.5,166.1,137.8,135.7,135.6,133.4,133.2,130.0,129.9,128.4,127.8,127.8,127.7,72.9,68.2,66.9,61.4$, 44.4, 41.3, 40.8, 31.8, 26.8, 19.0, 14.1; FAB MS m/z (relative intensity): 543 (7, $\mathrm{MH}^{+}$), 435 (3). Anal. Calcd. for $\mathrm{C}_{33} \mathrm{H}_{38} \mathrm{O}_{5}$ Si.0.3 $\mathrm{H}_{2} \mathrm{O}: \mathrm{C}, 72.37$; H, 7.10. Found: C, 72.12; H, 6.79. 


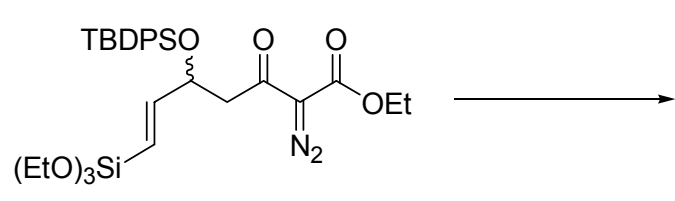

$( \pm)-7 \mathbf{c}$

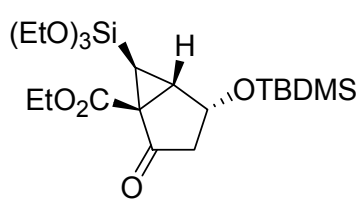

( \pm )-8c

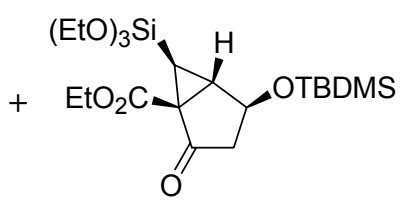

( \pm )-9c

Ethyl 2-oxo-4-(1,1,2,2-tetramethyl-1-silapropoxy)-6-(triethoxysilamethyl)bicyclo[3.1.0]hexanecarboxylate (( 1$)-8 \mathrm{c})$. Compound ( \pm )-7c $(777 \mathrm{mg}, 0.23 \mathrm{mmol})$ was treated as described for compound $( \pm)-7 \mathbf{b}$. The reaction mixture was stirred at the indicated temperature for $22 \mathrm{~h} .{ }^{1} \mathrm{H}-\mathrm{NMR}$ of the crude product showed a mixture of $( \pm)-8 \mathrm{c}$ and $( \pm)-9 \mathrm{c}$ epimers in a 4 to 1 ratio. After column chromatography purification (hexanes:ethyl acetate, 19:1), $80 \mathrm{mg}(62 \%$ yield) of pure compound ( \pm )-8c as a colorless oil: IR (neat): $1749 \mathrm{~cm}^{-1} ;{ }^{1} \mathrm{H}$ NMR $\delta 4.75(\mathrm{~m}, 1 \mathrm{H}), 4.18$ (q, $\left.J=7.2 \mathrm{~Hz}, 2 \mathrm{H}\right), 3.85$ (m, $\left.6 \mathrm{H}\right), 2.74(\mathrm{dd}, J=6.4,4.9 \mathrm{~Hz}$ $1 \mathrm{H}), 2.44(\mathrm{dd}, J=18.3,8.4 \mathrm{~Hz}, 1 \mathrm{H}), 2.21(\mathrm{dd}, J=18.3,6.8 \mathrm{~Hz}, 1 \mathrm{H}), 1.32(\mathrm{t}, J=7.0 \mathrm{~Hz}, 3 \mathrm{H}), 1.22(\mathrm{t}, J=7.0 \mathrm{~Hz}, 9 \mathrm{H}), 0.96$ $(\mathrm{d}, J=6.4 \mathrm{~Hz}, 1 \mathrm{H}), 0.89(\mathrm{~s}, 9 \mathrm{H}), 0.10(\mathrm{~s}, 3 \mathrm{H}), 0.07(\mathrm{~s}, 3 \mathrm{H}) ;{ }^{13} \mathrm{C}$ NMR $\delta 204.2,166.3,66.1,61.3,58.9,45.7,41.2,36.4,25.7$, 18.1, 18.0, 14.0, 12.6, -4.7, -4.8; FAB MS m/z (relative intensity): 461 (2, $\mathrm{MH}^{+}$), 415 (100). HRFABMS calculated for $\mathrm{C}_{21} \mathrm{H}_{40} \mathrm{O}_{7} \mathrm{Si}_{2} \mathrm{~K}\left(\mathrm{M}+\mathrm{K}^{+}\right): \mathrm{m} / \mathrm{z}=499.195$; found: $\mathrm{m} / \mathrm{z}=499.197$. Compound $( \pm)-9 \mathrm{c}$ could not be characterized because it was unstable during chromatographic purification.

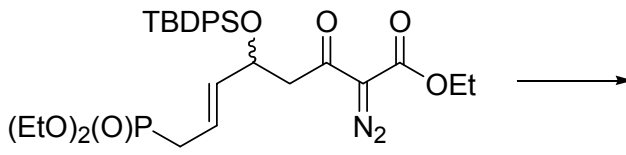

$( \pm)-7 d$

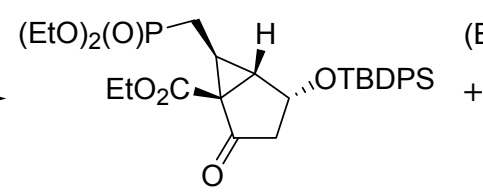

$( \pm)-8 d$

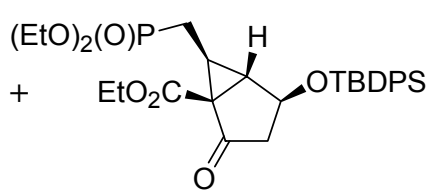

$( \pm)-9 d$

Ethyl 6-[(diethoxyphosphoryl)methyl]-4-(2,2-dimethyl-1,1-diphenyl-1-silapropoxy)-2-oxobicyclo[3.1.0]hexane carboxylate $(( \pm)-8 d$ and $( \pm)-9 d)$. Compound $( \pm)-7 \mathbf{d}(232 \mathrm{mg}, 0.39 \mathrm{mmol})$ was treated as described for compound $( \pm)-7 \mathbf{b}$. The reaction mixture was stirred at the indicated temperature for $22 \mathrm{~h}$. After column chromatography purification (hexanes:ethyl acetate, 1:1), $162 \mathrm{mg}$ of compound $( \pm)-8 d$ and $49 \mathrm{mg}$ of compound ( \pm )-9d (95 \% overall yield) were obtained as colorless oils. Compound ( \pm )-8d: IR (neat): $1741 \mathrm{~cm}^{-1} ;{ }^{1} \mathrm{H}$ NMR $\delta 7.69(\mathrm{~m}, 4 \mathrm{H}), 7.41(\mathrm{~m}, 6 \mathrm{H}), 4.63(\mathrm{dt}, J=8.1,5.4 \mathrm{~Hz}, 1 \mathrm{H}), 4.16(\mathrm{~m}, 6 \mathrm{H})$, $2.58(\mathrm{t}, J=5.4 \mathrm{~Hz}, 1 \mathrm{H}), 2.37(\mathrm{~m}, 1 \mathrm{H}), 2.23(\mathrm{ddd}, J=18.3,15.5,6.8 \mathrm{~Hz}, 1 \mathrm{H}), 2.21(\mathrm{~m} A B, 2 \mathrm{H}), 1.99(\mathrm{ddd}, J=19.3,15.5,7.2$ $\mathrm{Hz}, 1 \mathrm{H}), 1.33(\mathrm{~m}, 6 \mathrm{H}), 1.26(\mathrm{t}, J=7.1 \mathrm{~Hz}, 3 \mathrm{H}), 1.08(\mathrm{~s}, 9 \mathrm{H}) ;{ }^{13} \mathrm{C}$ NMR $\delta 201.7,165.8,135.6,133.2,130.0,127.8,66.6,62.2$ $(\mathrm{d}, J=6.6 \mathrm{~Hz}), 61.9(\mathrm{~d}, J=6.6 \mathrm{~Hz}), 61.6,45.5(\mathrm{~d}, J=8.7 \mathrm{~Hz}), 42.7,39.9(\mathrm{~d}, J=7.9 \mathrm{~Hz}), 26.8,26.1(\mathrm{~d}, J=2.3 \mathrm{~Hz}), 24.2(\mathrm{~d}, J=$ 144.6 Hz), 19.1, 16.5 (m), 14.2; FAB MS m/z (relative intensity): $573\left(84, \mathrm{MH}^{+}\right.$), 515 (75). Anal. Calcd. for $\mathrm{C}_{30} \mathrm{H}_{41} \mathrm{O}_{7} \mathrm{PSi}_{\mathrm{C}} \mathrm{C}_{\text {, }}$ 62.92; H, 7.22. Found: C, 62.79; H, 7.20. Compound ( \pm )-9d: IR (neat): $1742 \mathrm{~cm}^{-1} ;{ }^{1} \mathrm{H}$ NMR $\delta 7.63$ (m, 4 H), $7.41(\mathrm{~m}, 6 \mathrm{H})$, 
$4.44(\mathrm{~d}, J=5.0 \mathrm{~Hz}, 1 \mathrm{H}), 4.28(\mathrm{~m}, 2 \mathrm{H}), 3.99(\mathrm{~m}, 4 \mathrm{H}), 2.60(\mathrm{~d}, J=5.4 \mathrm{~Hz}, 1 \mathrm{H}), 2.34(\mathrm{dd}, J=19.0,5.0 \mathrm{~Hz}, 1 \mathrm{H}), 2.19(\mathrm{~d}, J=$ $19.0 \mathrm{~Hz}, 1 \mathrm{H}), 2.13$ (ddd, $J=18.2,15.4,6.0 \mathrm{~Hz}, 1 \mathrm{H}), 1.89$. (ddd, $J=19.1,15.4,9.0 \mathrm{~Hz}, 1 \mathrm{H}), 1.61$ (m, $1 \mathrm{H}), 1.34$ (t, $J=7.2 \mathrm{~Hz}$, $3 \mathrm{H}), 1.22(\mathrm{q}, J=6.9 \mathrm{~Hz}, 6 \mathrm{H}), 1.05(\mathrm{~s}, 9 \mathrm{H}) ;{ }^{13} \mathrm{C}$ NMR $\delta 204.0,166.3,135.7,133.3,133.1,130.0,129.9,127.8,127.7,68.2$, $62.0(\mathrm{~d}, J=6.6 \mathrm{~Hz}), 61.9$ (d, $J=6.3 \mathrm{~Hz}), 61.5,44.8,43.1,41.5(\mathrm{~d}, J=10.6 \mathrm{~Hz}), 26.8,24.0(\mathrm{~d}, J=142.0 \mathrm{~Hz}), 19.1,16.4(\mathrm{~m})$, 14.3; FAB MS m/z (relative intensity): $573\left(100, \mathrm{MH}^{+}\right), 527$ (52). Anal. Calcd. for $\mathrm{C}_{30} \mathrm{H}_{41} \mathrm{O}_{7} \mathrm{PSi}: \mathrm{C}, 62.92 ; \mathrm{H}, 7.22$. Found: $\mathrm{C}$, $62.65 ; \mathrm{H}, 7.32$.

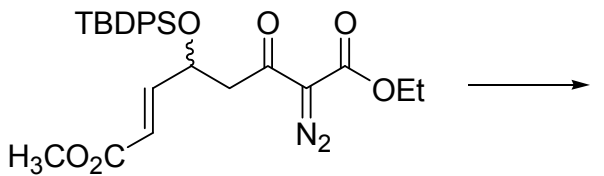

$( \pm)-7 f$

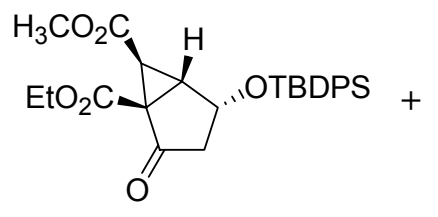

$( \pm)-8 f$

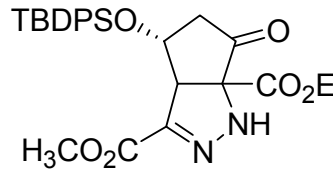

$( \pm)-10 f$

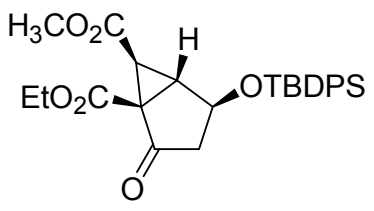

$( \pm)-9 f$

Ethyl 4-(2,2-dimethyl-1,1-diphenyl-1-silapropoxy)-6-(methoxycarbonyl)-2-oxobicyclo[3.1.0]hexanecarboxylate $(( \pm)-8 \mathrm{f}$ and ( \pm -9f) and methyl 6-(2,2-dimethyl-1,1-diphenyl-1-silapropoxy)-3a-(ethoxycarbonyl)-4-oxo-3,5,6,3a,6a-pentahydro2,3-diazapentalenecarboxylate ( $( \pm)-10 f$ and $( \pm)-11 f)$. Compound $( \pm)-7 \mathbf{f}(356 \mathrm{mg}, 0.70 \mathrm{mmol})$ was treated as described for compound $( \pm)-7 \mathbf{b}$. The reaction mixture was stirred at the indicated temperature for $48 \mathrm{~h}$. After column chromatography purification (hexanes:ethyl acetate, 4:1), four compounds were isolated: $30 \mathrm{mg}$ of compound ( \pm )-8f, $30 \mathrm{mg}$ of compound ( \pm )-9f, $184 \mathrm{mg}$ of compound $( \pm)-\mathbf{1 0 f}$ and $18 \mathrm{mg}$ of compound $( \pm)-\mathbf{1 1 f}$ (75 \% overall yield). Compound ( \pm )-8f: white solid; mp 147$148{ }^{\circ} \mathrm{C}$; IR (neat): $1746,1734 \mathrm{~cm}^{-1} ;{ }^{1} \mathrm{H}$ NMR $\delta$ 7.68-7.61 (m, $\left.4 \mathrm{H}\right), 7.48-7.36$ (m, $\left.6 \mathrm{H}\right), 4.74$ (ddd, $\left.J=8.5,7.1,5.1 \mathrm{~Hz}, 1 \mathrm{H}\right)$, $4.16(\mathrm{~m}, 2 \mathrm{H}), 3.76(s, 3 \mathrm{H}), 2.91(\mathrm{dd}, J=5.1,4.0 \mathrm{~Hz}), 2.70(\mathrm{~d}, J=4.0 \mathrm{~Hz}, 1 \mathrm{H}), 2.34(\mathrm{dd}, J=18.6,8.5 \mathrm{~Hz}, 1 \mathrm{H}), 2.14(\mathrm{dd}, J=$ 18.6, 7.1 Hz, $1 \mathrm{H}), 1.22(\mathrm{t}, J=7.1 \mathrm{~Hz}, 3 \mathrm{H}), 1.07(\mathrm{~s}, 9 \mathrm{H}) ;{ }^{13} \mathrm{C}$ NMR $\delta 201.6,168.4,163.7,135.6,133.0,132.8,130.2,130.1$, 128.0, 127.9, 66.5, 61.9, 52.6, 50.2, 41.3, 37.8, 27.8, 26.8, 19.1, 14.0; FAB MS m/z (relative intensity): $481\left(100, \mathrm{MH}^{+}\right), 435$ (53). Anal. Calcd. for $\mathrm{C}_{27} \mathrm{H}_{32} \mathrm{O}_{6} \mathrm{Si} .0 .2 \mathrm{H}_{2} \mathrm{O}$ : C, 67.03; H, 6.75. Found: C, 66.75; H, 6.79. Compound (土)-9f: colorless oil; IR (neat): 1755, $1733 \mathrm{~cm}^{-1} ;{ }^{1} \mathrm{H}$ NMR $\delta$ 7.71-7.61 (m, $\left.4 \mathrm{H}\right)$, 7.48-7.36 (m, $\left.6 \mathrm{H}\right), 4.46(\mathrm{~m}, 1 \mathrm{H}), 4.26(\mathrm{~m}, 2 \mathrm{H}), 3.67(s, 3 \mathrm{H}), 3.02(\mathrm{~d}$, 
$J=4.3 \mathrm{~Hz}), 2.20(\mathrm{mAB}, 2 \mathrm{H}), 2.03(\mathrm{~d}, J=4.3 \mathrm{~Hz}, 1 \mathrm{H}), 1.31(\mathrm{t}, J=7.1 \mathrm{~Hz}, 3 \mathrm{H}), 1.08(\mathrm{~s}, 9 \mathrm{H}) ;{ }^{13} \mathrm{C} \mathrm{NMR} \delta 203.9,167.7,164.2$, 135.7, 135.6, 133.0, 132.9, 130.0, 127.9, 127.8, 68.4, 61.8, 52.6, 45.8, 43.1, 39.8, 29.2, 26.7, 19.1, 14.1; FAB MS m/z (relative intensity): $481\left(30, \mathrm{MH}^{+}\right), 423$ (82). Anal Calcd. for $\mathrm{C}_{27} \mathrm{H}_{32} \mathrm{O}_{6} \mathrm{Si}$ : C, 67.47; H, 6.71. Found: C, 67.36; H, 6.56. Compound ( \pm )10f: colorless oil; IR (neat): 3349, 1764, 1737, $1703 \mathrm{~cm}^{-1}$; ${ }^{1} \mathrm{H}$ NMR $\delta$ 7.65-7.59 (m, $\left.4 \mathrm{H}\right), 7.48-7.36$ (m, $\left.6 \mathrm{H}\right), 7.22$ (bs, $1 \mathrm{H}$ ), $4.84(\mathrm{ddd}, J=6.1,5.1,2.3 \mathrm{~Hz}, 1 \mathrm{H}), 4.39$ (d, $J=6.1 \mathrm{~Hz}, 1 \mathrm{H}), 4.39$ (q, $J=7.1 \mathrm{~Hz}, 2 \mathrm{H}), 3.81(\mathrm{~s}, 3 \mathrm{H}), 2.38(\mathrm{dd}, J=17.2,5.1$ $\mathrm{Hz}, 1 \mathrm{H}), 2.27$ (dd, $\left.J=17.2,2.3 \mathrm{~Hz}, 1 \mathrm{H}), 1.23(\mathrm{t}, J=7.1 \mathrm{~Hz}, 3 \mathrm{H}), 0.97(\mathrm{~s}, 9 \mathrm{H}) ;{ }^{13} \mathrm{C} \mathrm{NMR} \delta 205.5,168.7,162.3,141.2\right)$, $135.8,133.1,132.2,130.1,130.0,127.8,80.6,69.8,63.0,59.4,52.2,48.1,26.5,19.1,14.0 ; \mathrm{FAB} \mathrm{MS} \mathrm{m} / \mathrm{z}$ (relative intensity): $509\left(20, \mathrm{MH}^{+}\right), 451$ (33). Anal. Calcd. for $\mathrm{C}_{27} \mathrm{H}_{32} \mathrm{~N}_{2} \mathrm{O}_{6} \mathrm{Si} .0 .4 \mathrm{H}_{2} \mathrm{O}: \mathrm{C}, 62.96 ; \mathrm{H}, 6.43 ; \mathrm{N}, 5.22$. Found: C, 63.16; H, 6.42; N, 4.97. Compound (土)-11f: colorless oil; IR (neat): 3344, 1764, 1736, $1702 \mathrm{~cm}^{-1} ;{ }^{1} \mathrm{H}$ NMR $\delta$ 7.74-7.63 (m, $\left.4 \mathrm{H}\right), 7.50-7.36$ (m, 6 H), $7.11(\mathrm{bs}, 1 \mathrm{H}), 4.68(\mathrm{~m}, 1 \mathrm{H}), 4.45(\mathrm{bs}, 1 \mathrm{H}), 4.35(\mathrm{~m}, 2 \mathrm{H}), 3.75(\mathrm{~s}, 3 \mathrm{H}), 2.25(\mathrm{~m} A B, 2 \mathrm{H}), 1.33$ (t, J= 7.1 Hz, $3 \mathrm{H}), 1.09$ (s, $9 \mathrm{H}) ;{ }^{13} \mathrm{C}$ NMR $\delta 204.2,167.9,161.3,140.1,135.9,135.7,133.1,132.5,130.1,127.8,78.2,71.6,62.9,52.3,45.3,26.7,19.2$, 14.2; FAB MS m/z (relative intensity): $509\left(20, \mathrm{MH}^{+}\right), 451$ (100). Anal. Calcd. for $\mathrm{C}_{27} \mathrm{H}_{32} \mathrm{~N}_{2} \mathrm{O}_{6} \mathrm{Si}: \mathrm{C}, 63.96 ; \mathrm{H}, 6.34$. Found: C, $64.23 ; \mathrm{H}, 6.36$.

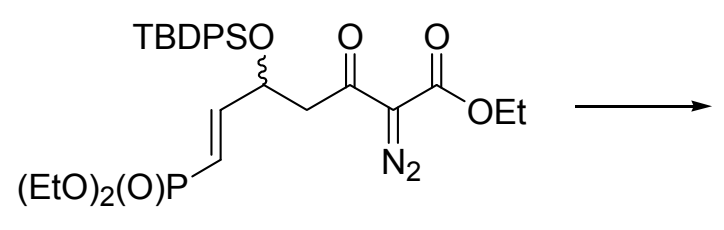

$( \pm)-7 e$

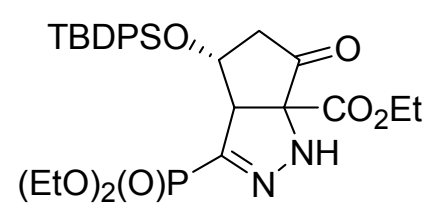

$( \pm)-10 e$

\section{Ethyl 3-(diethoxyphosphoryl)-4-(2,2-dimethyl-1,1-diphenyl-1-silapropoxy)-6-oxo-1,4,5,3a,6a-pentahydro-1,2-}

diazapentalen-6a-carboxylate $(( \pm)-10 \mathrm{e})$. Compound $( \pm)-7 \mathrm{e}(103 \mathrm{mg}, 0.18 \mathrm{mmol})$ was treated as described for compound $( \pm)-$

7b. The reaction mixture was stirred at the indicated temperature for $48 \mathrm{~h}$. After column chromatography purification (hexanes:ethyl acetate, 1:1), only $26 \mathrm{mg}$ (25\% yield) of the mayor product $( \pm)$-10e could be isolated and characterized: colorless oil; IR (neat): 3347, 1750, 1733, $1022 \mathrm{~cm}^{-1} ;{ }^{1} \mathrm{H}$ NMR $\delta$ 7.77-7.62 (m, $\left.4 \mathrm{H}\right), 7.45-7.35$ (m, $\left.6 \mathrm{H}\right), 7.21$ (bs, $\left.1 \mathrm{H}\right), 4.81$ (ddd, $J=7.0,4.8,2.3 \mathrm{~Hz}, 1 \mathrm{H}), 4.26(\mathrm{~d}, J=7.0 \mathrm{~Hz}, 1 \mathrm{H}), 4.18(\mathrm{~m}, 6 \mathrm{H}), 2.34(\mathrm{dd}, J=17.0,4.5 \mathrm{~Hz}, 1 \mathrm{H}), 2.26(\mathrm{dd}, J=17.0,2.2$ $\mathrm{Hz}, 1 \mathrm{H}), 1.30(\mathrm{~m}, 6 \mathrm{H}), 1.20$ (t, $J=7.1 \mathrm{~Hz}, 3 \mathrm{H}), 1.04$ (s, $9 \mathrm{H}) ;{ }^{13} \mathrm{C}$ NMR $\delta$ 205.6, 169.1, 142.0, 136.1, 135.9, 133.7, 132.4, 129.9, 127.7, 80.6, 70.4, 63.0, 62.9, $62.8(\mathrm{~d}, \mathrm{~J}=6.1 \mathrm{~Hz}), 48.6,26.7,19.2,16.3(\mathrm{~d}, J=6.1 \mathrm{~Hz}), 13.9$; FAB MS m/z (relative intensity): $587\left(34, \mathrm{MH}^{+}\right), 509$ (100). HRFABMS calculated for $\mathrm{C}_{29} \mathrm{H}_{40} \mathrm{~N}_{2} \mathrm{O}_{7} \mathrm{PSi}\left(\mathrm{MH}^{+}\right): \mathrm{m} / \mathrm{z}=587.234$; found: $\mathrm{m} / \mathrm{z}=587.236$. 


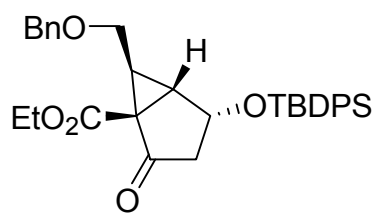

$( \pm)-8 b$

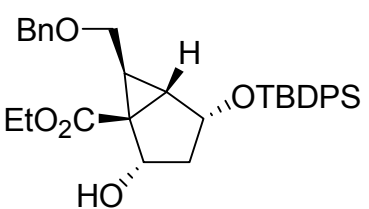

$( \pm)-12$

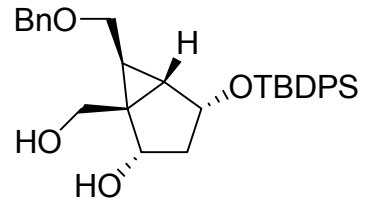

$( \pm)-13$

Ethyl 4-(2,2-dimethyl-1,1-diphenyl-1-silapropoxy)-2-hydroxy-6-[(phenylmethoxy)methyl]bicyclo[3.1.0]hexane

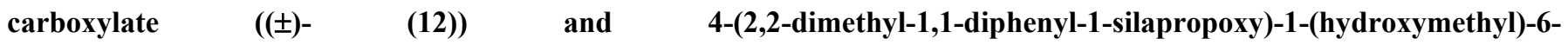
[(phenylmethoxy)methyl]bicyclo[3.1.0]hexan-2-ol (( \pm )-(13)). A stirred solution of bicyclo compound ( \pm )-8b (5.33 g, 9.82 mmol) in methanol $(50 \mathrm{~mL})$ and dichloromethane $(25 \mathrm{~mL})$ was cooled to $-25{ }^{\circ} \mathrm{C}$ and treated with $\mathrm{NaBH}_{4}(450 \mathrm{mg}, 11.80$ mmol). After further stirring between -25 and $-15{ }^{\circ} \mathrm{C}$ for $30 \mathrm{~min}$, the temperature was lowered to $-20{ }^{\circ} \mathrm{C}$ and the reaction was quenched with $\mathrm{AcOH}$ until neutral $\mathrm{pH}$. The mixture was concentrated and the residue was dissolved in dichloromethane (250 $\mathrm{mL})$. The organic phase was washed with brine $(50 \mathrm{~mL})$, dried $\left(\mathrm{MgSO}_{4}\right)$ and concentrated. This compound could be used directly in the next step without further purification; however, an analytical sample was obtained following flash column chromatography (silica gel) employing hexanes:ethyl acetate (9:1) as eluant to give ( \pm )-12 as a clear oil. A stirred suspension of LAH (1.76 g, $46.15 \mathrm{mmol})$ in diethyl ether $(130 \mathrm{~mL})$ was treated dropwise with a solution of crude $( \pm)-12$ (ca. $9.82 \mathrm{mmol})$ in diethyl ether $(20 \mathrm{~mL})$ at $0{ }^{\circ} \mathrm{C}$. After stirring for $2 \mathrm{~h}$, water $(2.0 \mathrm{~mL}), 15 \%$ aqueous $\mathrm{NaOH}$ solution $(2.0 \mathrm{~mL})$, and water $(2.6 \mathrm{~mL})$ were successively added dropwise at $0{ }^{\circ} \mathrm{C}$. The reaction mixture was filtered through a path of Celite and the filtrate was concentrated under vacuum. The residue was purified by flash column chromatography (silica gel) using hexanes:ethyl acetate (3:2) as eluant to afford $3.67 \mathrm{~g}\left(75 \%\right.$, two steps) of $( \pm)-13$ as a clear oil. Compound $( \pm)-12$ : IR (neat): $3446,1717 \mathrm{~cm}^{-1} ;{ }^{1} \mathrm{H}$ NMR $\delta$ 7.68-7.62 (m, 4 H), 7.42-7.32 (m, $11 \mathrm{H}), 4.56(A B \mathrm{q}, J=11.9 \mathrm{~Hz}, 2 \mathrm{H}), 4.45(\mathrm{t}, J=8.5 \mathrm{~Hz}, 1 \mathrm{H}), 4.33(\mathrm{~m}, 1 \mathrm{H}), 4.08(\mathrm{q}$, $J=7.1 \mathrm{~Hz}, 2 \mathrm{H}), 3.83(\mathrm{dd}, J=10.6,5.5 \mathrm{~Hz}, 1 \mathrm{H}), 3.49(\mathrm{dd}, J=10.6,8.6 \mathrm{~Hz}, 1 \mathrm{H}), 2.38(\mathrm{dt}, J=8.6,5.5 \mathrm{~Hz}, 1 \mathrm{H}), 2.35(\mathrm{dt}, J=$ 13.6, $7.8 \mathrm{~Hz}, 1 \mathrm{H}), 1.93(\mathrm{t}, J=5.5 \mathrm{~Hz}, 1 \mathrm{H}), 1.45(\mathrm{dt}, J=13.6,8.8 \mathrm{~Hz}, 1 \mathrm{H}), 1.18(\mathrm{t}, J=7.1 \mathrm{~Hz}, 3 \mathrm{H}), 1.03(\mathrm{~s}, 9 \mathrm{H}) ;{ }^{13} \mathrm{C} \mathrm{NMR} \delta$ $171.9,138.5,135.6,133.9,133.7,133.2,129.8,129.7,128.3,127.7,127.6,127.5,127.4,72.3,70.1,70.0,67.3,60.9,39.3,36.9$, 36.8, 26.8, 24.0, 19.1, 14.2; FAB MS m/z (relative intensity): 545 (2, $\left.\mathrm{MH}^{+}\right), 437$ (2). Anal. Calcd. for $\mathrm{C}_{33} \mathrm{H}_{40} \mathrm{O}_{5} \mathrm{Si}: \mathrm{C}, 72.76 ; \mathrm{H}$, 7.40. Found: C, 72.90; H, 7.34. Compound ( \pm )-13: IR (neat): 3392, $1721 \mathrm{~cm}^{-1} ;{ }^{1} \mathrm{H}$ NMR $\delta$ 7.66-7.62 (m, $\left.4 \mathrm{H}\right), 7.44-7.30$ (m, 11 H), $4.57(A B \mathrm{q}, J=11.7 \mathrm{~Hz}, 2 \mathrm{H}), 4.29(\mathrm{~m}, 2 \mathrm{H}), 3.97(\mathrm{dd}, J=10.5,5.6 \mathrm{~Hz}, 1 \mathrm{H}), 3.82(\mathrm{~d}, J=12.2 \mathrm{~Hz}, 1 \mathrm{H}), 3.49(\mathrm{bs}, 1 \mathrm{H}$, this signal changes to a doublet with $\mathrm{D}_{2} \mathrm{O}, J=12.2 \mathrm{~Hz}$ ), 3.04 (t, $\left.J=10.6 \mathrm{~Hz}, 1 \mathrm{H}\right), 2.43(\mathrm{bs}, 1 \mathrm{H}), 2.15(\mathrm{dt}, J=13.5,7.7 \mathrm{~Hz}, 1 \mathrm{H})$, $2.06(\mathrm{ddd}, J=10.6,5.6,3.5 \mathrm{~Hz}, 1 \mathrm{H}), 1.39(\mathrm{dt}, J=13.5,8.7 \mathrm{~Hz}, 1 \mathrm{H}), 1.17(\mathrm{~m}, 1 \mathrm{H}), 1.03(\mathrm{~s}, 9 \mathrm{H}) ;{ }^{13} \mathrm{C}$ NMR $\delta$ 137.3, 135.6, 134.3, 133.9, 129.7, 129.6, 128.6, 128.0, 127.6, 127.5, 75.4, 73.1, 70.8, 69.9, 65.2, 39.5, 38.0, 34.3, 26.8, 19.1, 18.1; FAB MS m/z (relative intensity): $503\left(2, \mathrm{MH}^{+}\right), 395$ (1). Anal. Calcd. for $\mathrm{C}_{31} \mathrm{H}_{38} \mathrm{O}_{4} \mathrm{Si}$ : C, 74.06; H, 7.62. Found: $\mathrm{C}, 73.91 ; \mathrm{H}, 7.68$. 


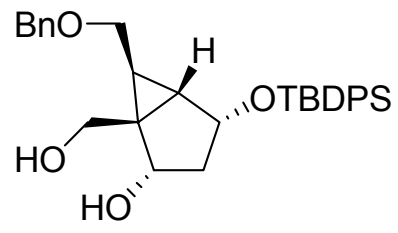

$( \pm)-13$

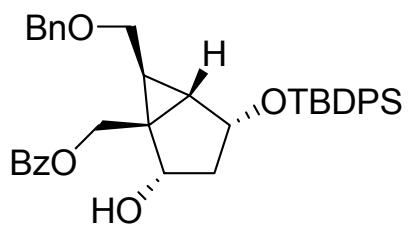

$( \pm)-14$

\{4-(2,2-Dimethyl-1,1-diphenyl-1-silapropoxy)-2-hydroxy-6-[(phenylmethoxy)methyl]bicyclo[3.1.0]hexyl\}methyl benzoate

(( \pm )-14). Triethylamine $(1.5 \mathrm{~mL}, 10.70 \mathrm{mmol})$ and $\mathrm{BzCl}(1.0 \mathrm{~mL}, 8.59 \mathrm{mmol})$ were added to a solution of compound $( \pm)-\mathbf{1 3}$ $(3.60 \mathrm{~g}, 7.16 \mathrm{mmol})$ in anhydrous acetonitrile $(260 \mathrm{~mL})$ at $0{ }^{\circ} \mathrm{C}$. The reaction mixture was stirred at the same temperature for 3 $\mathrm{h}$ and at room temperature for $20 \mathrm{~min}$. The reaction was quenched by addition of saturated solution of $\mathrm{NH}_{4} \mathrm{Cl}(100 \mathrm{~mL})$. The mixture was extracted with ethyl acetate $(3 \times 200 \mathrm{~mL})$ and the combined organic phases were washed with saturated solution of $\mathrm{NaHCO}_{3}(2 \times 40 \mathrm{~mL})$, dried $\left(\mathrm{MgSO}_{4}\right)$ and concentrated in vacuum. The residue was purified by flash column chromatography (silica gel) employing hexanes : ethyl acetate (17:3) as eluting system to afford $1.33 \mathrm{~g}$ (80 \% yield) of compound ( \pm )-14 as a colorless oil: IR (neat): 3511, $1715 \mathrm{~cm}^{-1} ;{ }^{1} \mathrm{H}$ NMR $\delta 7.91$ (m, $\left.2 \mathrm{H}\right)$, 7.66-7.59 (m, $\left.4 \mathrm{H}\right), 7.51$ (m, $\left.1 \mathrm{H}\right), 7.40-7.25$ (m, $\left.13 \mathrm{H}\right), 4.53$ $(A B \mathrm{q}, J=11.9 \mathrm{~Hz}, 2 \mathrm{H}), 4.49(\mathrm{~d}, J=12.2 \mathrm{~Hz}, 1 \mathrm{H}), 4.35(\mathrm{~d}, J=12.2 \mathrm{~Hz}, 1 \mathrm{H}), 4.30(\mathrm{~m}, 1 \mathrm{H}), 4.25(\mathrm{t}, J=8.4 \mathrm{~Hz}, 1 \mathrm{H}), 3.71(\mathrm{dd}$, $J=10.6,5.9 \mathrm{~Hz}, 1 \mathrm{H}), 3.39(\mathrm{dd}, J=10.6,8.2 \mathrm{~Hz}, 1 \mathrm{H}), 2.14(\mathrm{dt}, J=13.4,7.7 \mathrm{~Hz}, 1 \mathrm{H}), 2.01(\mathrm{ddd}, J=8.2,5.9,3.9 \mathrm{~Hz}, 1 \mathrm{H})$, 1.78 (bs, $1 \mathrm{H}), 1.37$ (m, $\left.2 \mathrm{H}, \mathrm{H}-3_{\mathrm{b}}\right), 1.00$ (s, $\left.9 \mathrm{H}\right) ;{ }^{13} \mathrm{C}$ NMR $\delta$ 166.9, 138.2, 135.7, 135.6, 134.2, 133.0, 130.0, 129.7, 129.6, $128.4,128.3,127.7,127.6,127.5,72.8,72.5,70.6,68.6,65.1,38.2,36.6,33.8,26.8,19.3,19.1 ; \mathrm{FAB} \mathrm{MS} \mathrm{m} / \mathrm{z}$ (relative intensity): $607\left(3, \mathrm{MH}^{+}\right), 549$ (2). Anal. Calcd. for $\mathrm{C}_{38} \mathrm{H}_{42} \mathrm{O}_{5} \mathrm{Si}$ : C, 75.21; H, 6.98. Found: $\mathrm{C}, 75.21 ; \mathrm{H}, 7.26$.

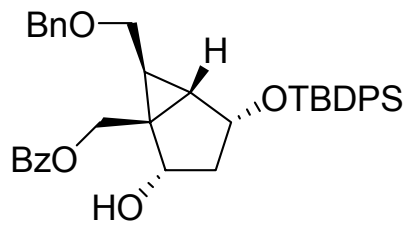

$( \pm)-14$

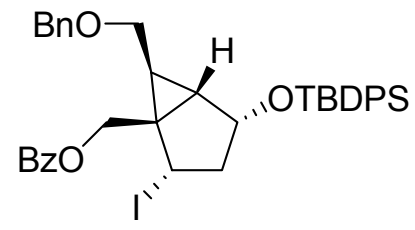

$( \pm)-15$

\{4-(2,2-Dimethyl-1,1-diphenyl-1-silapropoxy)-2-iodo-6-[(phenylmethoxy)methyl]bicyclo[3.1.0]hexyl\}methyl benzoate

$(( \pm)-15)$. A solution of $( \pm)-14(3.45 \mathrm{~g}, 5.69 \mathrm{mmol})$ in toluene $(150 \mathrm{~mL})$ was treated with triphenylphosphine $(2.98 \mathrm{~g}, 11.37$ mmol), imidazole $(1.16 \mathrm{~g}, 17.07 \mathrm{mmol})$ and iodine $(2.16 \mathrm{~g}, 8.54 \mathrm{mmol})$. The mixture was heated at $100{ }^{\circ} \mathrm{C}$ for $1 \mathrm{~h}$ under a blanket or argon. After reaching room temperature, the mixture was poured into a saturated solution of $\mathrm{NaHCO}_{3}(100 \mathrm{~mL})$. Excess triphenylphosphine was destroyed by the addition of iodine until the iodine coloration persisted in the organic layer. The organic phase was washed with $5 \%(\mathrm{wt}) \mathrm{Na}_{2} \mathrm{~S}_{2} \mathrm{O}_{3}(2 \times 30 \mathrm{~mL})$ and brine $(30 \mathrm{~mL})$, dried $\left(\mathrm{MgSO}_{4}\right)$ and concentrated in vacuo. 
The residue was purified by flash column chromatography (silica gel) employing hexanes:ethyl acetate (19:1) as eluting system to afford $3.6 \mathrm{~g}$ (88 \% yield) of compound $( \pm)-15$ as a colorless oil: IR (neat): $1715 \mathrm{~cm}^{-1} ;{ }^{1} \mathrm{H}$ NMR $\delta 7.91(\mathrm{~m}, 2 \mathrm{H}), 7.66-7.59$ (m, $4 \mathrm{H}), 7.52(\mathrm{~m}, 1 \mathrm{H}), 7.40-7.25(\mathrm{~m}, 13 \mathrm{H}), 4.66(\mathrm{~d}, J=11.8 \mathrm{~Hz}, 1 \mathrm{H}), 4.53(\mathrm{~d}, J=11.8 \mathrm{~Hz}, 1 \mathrm{H}), 4.51(\mathrm{~d}, J=12.6 \mathrm{~Hz}, 1 \mathrm{H})$, $4.39(\mathrm{~m}, 1 \mathrm{H}), 4.34(\mathrm{dd}, J=10.3,7.9 \mathrm{~Hz}, 1 \mathrm{H}), 4.28(\mathrm{~d}, J=12.6 \mathrm{~Hz}, 1 \mathrm{H}), 3.72(\mathrm{dd}, J=11.0,5.8 \mathrm{~Hz}, 1 \mathrm{H}), 3.41(\mathrm{dd}, J=11.0$, $8.1 \mathrm{~Hz}, 1 \mathrm{H}), 2.45(\mathrm{dt}, J=15.0,7.9 \mathrm{~Hz}, 1 \mathrm{H}), 1.93(\mathrm{ddd}, J=15.0,10.3,8.2 \mathrm{~Hz}, 1 \mathrm{H}), 1.83-1.75(\mathrm{~m}, 2 \mathrm{H}), 1.03(\mathrm{~s}, 9 \mathrm{H}) ;{ }^{13} \mathrm{C}$ NMR $\delta 166.3,138.2,135.6,135.5,133.8,133.6,133.0,129.9,129.8,129.6,128.4,128.3,127.7,127.6,127.5,73.1,72.5,68.1$, 63.7, 43.3, 39.1, 38.5, 28.6, 26.8, 25.8, 19.1; FAB MS m/z (relative intensity): 717 (2, $\left.\mathrm{MH}^{+}\right), 659$ (2). Anal. Calcd. for $\mathrm{C}_{38} \mathrm{H}_{41} \mathrm{IO}_{4} \mathrm{Si} .:$ C, 63.98; H, 5.77. Found: C, 64.43; H, 5.92.

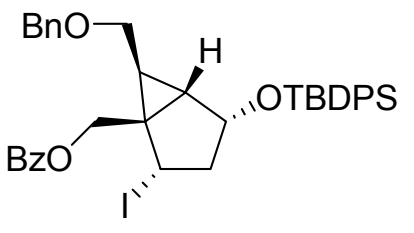

$( \pm)-15$

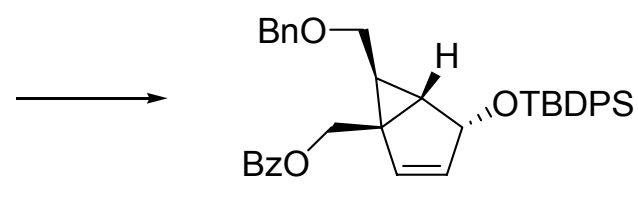

$( \pm)-16$

\section{\{4-(2,2-Dimethyl-1,1-diphenyl-1-silapropoxy)-6-[(phenylmethoxy)methyl]bicyclo[3.1.0]hex-2-enyl\}methyl benzoate $(( \pm)-$}

16). A mixture of ( \pm )-15 (2.38 g, $1.15 \mathrm{mmol})$ and 1,8-diazabicyclo[5.4.0]undec-7-ene (DBU, $5.0 \mathrm{~mL})$ in toluene $(50 \mathrm{~mL})$ was heated at $100{ }^{\circ} \mathrm{C}$ for $9 \mathrm{~h}$ under argon gas. After reaching room temperature, water was added $(10 \mathrm{~mL})$ and the organic layer was washed twice with brine $(2 \times 10 \mathrm{~mL})$. The organic phase was dried $\left(\mathrm{MgSO}_{4}\right)$ and the residue was purified by flash column chromatography (silica gel) employing hexane:ethyl acetate (49:1) as eluting system to afford $976 \mathrm{mg}$ (50\% yield) of compound ( \pm )-16 as a colorless oil: IR (neat): $1717 \mathrm{~cm}^{-1}$; ${ }^{1} \mathrm{H}$ NMR $\delta 7.90(\mathrm{~m}, 2 \mathrm{H}), 7.64-7.60(\mathrm{~m}, 4 \mathrm{H}), 7.45(\mathrm{~m}, 1 \mathrm{H}), 7.35-$ $7.15(\mathrm{~m}, 13 \mathrm{H}), 4.97(\mathrm{~d}, J=5.5 \mathrm{~Hz}, 1 \mathrm{H}), 5.20(\mathrm{~m}, 2 \mathrm{H}), 4.51(\mathrm{~d}, J=12.3 \mathrm{~Hz}, 1 \mathrm{H}), 4.44(A B \mathrm{q}, J=12.1 \mathrm{~Hz}, 2 \mathrm{H}), 4.32(\mathrm{~d}, J=$ $12.3 \mathrm{~Hz}, 1 \mathrm{H}), 3.64$ (dd, $J=10.7,5.3 \mathrm{~Hz}, 1 \mathrm{H}), 3.15(\mathrm{dd}, J=10.7,9.2 \mathrm{~Hz}, 1 \mathrm{H}), 1.73(\mathrm{~m}, 1 \mathrm{H}), 1.29(\mathrm{~m}, 1 \mathrm{H}), 0.98(\mathrm{~s}, 9 \mathrm{H}) ;{ }^{13} \mathrm{C}$ NMR $\delta 166.1,138.2,137.3,135.8,135.7,134.2,134.1,132.8,132.4,130.3,129.7,129.6,128.3,128.2,127.7,127.6,127.5$, 78.6, 72.7, 69.1, 63.8, 39.4, 33.5, 27.7, 26.9, 19.2; FAB MS m/z (relative intensity): $588\left(4, \mathrm{MH}^{+}\right)$. Anal.. Calcd. for $\mathrm{C}_{38} \mathrm{H}_{40} \mathrm{O}_{4} \mathrm{Si}: \mathrm{C}, 77.51 ; \mathrm{H}, 6.85$. Found: $\mathrm{C}, 77.58 ; \mathrm{H}, 6.91$. 


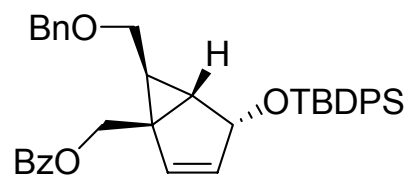

$( \pm)-16$

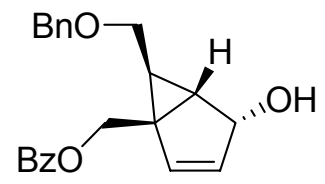

$( \pm)-17$

\{4-Hydroxy-6-[(phenylmethoxy)methyl]bicyclo[3.1.0]hex-2-enyl\}methyl benzoate $(( \pm)-17)$. A solution of $( \pm)-16(758 \mathrm{mg}$

$1.26 \mathrm{mmol})$ in anhydrous tetrahydrofuran $(50 \mathrm{~mL})$ was treated dropwise with $1 \mathrm{M}$ solution of tetrabutylammonium fluoride in tetrahydrofuran $(2.0 \mathrm{~mL})$. The reaction mixture was stirred at room temperature for $1.5 \mathrm{~h}$. The volatiles were removed in vacuo and the residue was purified by flash column chromatography (silica gel) employing hexane:ethyl acetate (7:3) as eluting system to afford $436 \mathrm{mg}$ (99 \% yield) of compound ( \pm )-17 as a colorless oil: IR (neat): $3349,3268,1720 \mathrm{~cm}^{-1} ;{ }^{1} \mathrm{H}$ NMR $\delta 8.00$ $(\mathrm{m}, 2 \mathrm{H}), 7.52(\mathrm{~m}, 1 \mathrm{H}), 7.40(\mathrm{~m}, 2 \mathrm{H}), 7.27(\mathrm{~m}, 5 \mathrm{H}), 6.08(\mathrm{~d}, J=5.5 \mathrm{~Hz}, 1 \mathrm{H}), 5.33(\mathrm{~d}, J=5.5 \mathrm{~Hz}, 1 \mathrm{H}), 5.24(\mathrm{~d}, J=6.4 \mathrm{~Hz}, 1$ H), $4.60(\mathrm{~d}, J=12.2 \mathrm{~Hz}, 1 \mathrm{H}), 4.48(\mathrm{~d}, J=12.2 \mathrm{~Hz}, 1 \mathrm{H}), 4.47(A B \mathrm{q}, J=11.8 \mathrm{~Hz}, 2 \mathrm{H}), 3.68(\mathrm{dd}, J=10.6,5.8 \mathrm{~Hz}, 1 \mathrm{H}), 3.35$ $(\mathrm{dd}, J=10.6,8.6 \mathrm{~Hz}, 1 \mathrm{H}), 1.73(\mathrm{dd}, J=6.4,3.9 \mathrm{~Hz}, 1 \mathrm{H}), 1.52(\mathrm{ddd}, J=8.6,5.8,3.9 \mathrm{~Hz}, 1 \mathrm{H}) ;{ }^{13} \mathrm{C}$ NMR $\delta 166.6,138.4$, 137.9, 132.9, 132.1, 130.2, 129.6, 128.4, 128.3, 127.4, 127.7, 77.7, 72.9, 68.9, 63.6, 39.7, 33.4, 28.2; FAB MS m/z (relative intensity): $351\left(58, \mathrm{MH}^{+}\right), 333$ (22). Anal. Calcd. for $\mathrm{C}_{22} \mathrm{H}_{22} \mathrm{O}_{4}$ : C, 75.41; H, 6.33. Found: C, 75.17; H, 6.48.

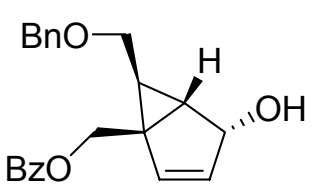

$( \pm)-17$

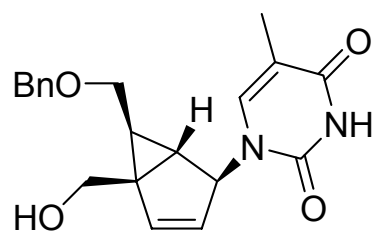

$( \pm)-18$

\section{1-\{5-(Hydroxymethyl)-6-[(phenylmethoxy)methyl]bicyclo[3.1.0]hex-3-en-2-yl\}-5-methyl-1,3-dihydropyrimidine-2,4-}

dione (( \pm )-18). A solution of triphenylphosphine $(704 \mathrm{mg}, 2.68 \mathrm{mmol})$ in anhydrous THF $(20 \mathrm{~mL})$ was treated dropwise with diisopropyl azodicarboxylate $(0.50 \mathrm{~mL}, 2.58 \mathrm{mmol})$ at $0{ }^{\circ} \mathrm{C}$. After the mixture was stirred for $30 \mathrm{~min}$ at the above mentioned temperature, $N^{3}$-benzoylthimine $(360 \mathrm{mg}, 1.56 \mathrm{mmol})$ and a THF solution $(10 \mathrm{~mL})$ of $( \pm)-17(360 \mathrm{mg}, 1.05 \mathrm{mmol})$ were successively added. The reaction mixture was stirred at $0{ }^{\circ} \mathrm{C}$ for $2 \mathrm{~h}$ and then was allowed to reach room temperature overnight. All volatiles were removed in vacuo and the residue was purified by flash column chromatography (silica gel) employing hexane:ethyl acetate (4:1) as eluant to afford $280 \mathrm{mg}$ of intermediate which was used without characterization in the next step. $217 \mathrm{mg}(0.38 \mathrm{mmol})$ of crude product was dissolved in methanolic ammonia $(8 \mathrm{~mL})$ in a sealed tube. The solution was stirred at $60{ }^{\circ} \mathrm{C}$ for $15 \mathrm{~h}$. Volatiles were removed in vacuo and the residue was purified by flash column chromatography (silica gel) 
employing hexane:ethyl acetate (1:1) as eluant to afford $111 \mathrm{mg}$ (37\% yield, both steps) of compound ( \pm )-18 as a white foam: IR (neat): 3396, 3149, $1671 \mathrm{~cm}^{-1}$; ${ }^{1} \mathrm{H}$ NMR $\delta 8.40$ (br s, $\left.1 \mathrm{H}\right), 7.35$ (m, $\left.5 \mathrm{H}\right), 6.88(\mathrm{~d}, J=1.3 \mathrm{~Hz}, 1 \mathrm{H}), 6.63(\mathrm{dm} .1 \mathrm{H}), 5.48$ (dist t, $1 \mathrm{H}), 5.39$ (dt, $J=5.5,1.9 \mathrm{~Hz}, 1 \mathrm{H}), 4.58(A B q, J=11.7 \mathrm{~Hz}, 2 \mathrm{H}), 3.89(\mathrm{~d}, J=12.3 \mathrm{~Hz}, 1 \mathrm{H}), 3.89$ (dd, $J=10.7,5.0 \mathrm{~Hz}$, $1 \mathrm{H}), 3.77(\mathrm{~d}, J=12.3 \mathrm{~Hz}, 1 \mathrm{H}), 3.13(\mathrm{t}, J=10.7 \mathrm{~Hz}, 1 \mathrm{H}), 1.88(\mathrm{~d}, J=1.3 \mathrm{~Hz}, 3 \mathrm{H}), 1.60(\mathrm{~m}, 1 \mathrm{H}), 1.17(\mathrm{ddd}, J=10.7,5.0,3.8$ $\mathrm{Hz}, 1 \mathrm{H}) ;{ }^{13} \mathrm{C}$ NMR $\delta 163.4,150.6,143.9,137.0,136.7,128.6,128.2,128.1,126.0,111.0,73.4,69.1,61.0,60.2,43.3,35.9$, 33.2, 12.5; FAB MS m/z (relative intensity): $355\left(62, \mathrm{MH}^{+}\right), 337$ (18). Anal. Calcd. for $\mathrm{C}_{20} \mathrm{H}_{22} \mathrm{~N}_{2} \mathrm{O}_{4} \cdot 0.5 \mathrm{H}_{2} \mathrm{O}: \mathrm{C}, 66.10 ; \mathrm{H}, 6.38$; N, 7.71. Found: C, 66.18; H, 6.42; N, 7.44.

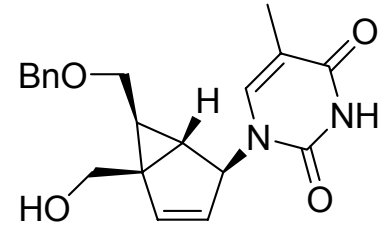

$( \pm)-18$

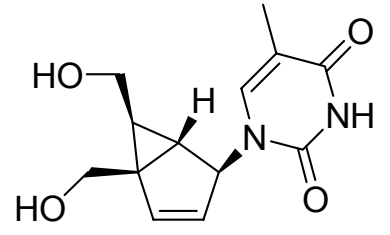

$( \pm)-1$

1-[5,6-Bis(hydroxymethyl)bicyclo[3.1.0]hex-3-en-2-yl]-5-methyl-1,3-dihydropyrimidine-2,4-dione (( \pm )-1). A solution of ( \pm )-18 (105 mg, $0.25 \mathrm{mmol})$ in anhydrous dichloromethane $(10 \mathrm{~mL})$ was treated with boron trichloride $\left(1 \mathrm{M}^{\text {in }} \mathrm{CH}_{2} \mathrm{Cl}_{2}, 2.5\right.$ $\mathrm{mL}$ ) at $-78{ }^{\circ} \mathrm{C}$ under a blanket of argon. The reaction mixture was stirred at the same temperature for $4 \mathrm{~h}$ and at $-20{ }^{\circ} \mathrm{C}$ for $1 \mathrm{~h}$. Methanol $(2 \mathrm{~mL})$ was carefully added and the mixture was allowed to reach room temperature. All volatiles were removed in vacuo and the residue was co-evaporated with methanol $(4 \times 5 \mathrm{~mL})$. The residue was purified by flash column chromatography (silica gel) employing $\mathrm{CHCl}_{3}$ :methanol (49:1) as eluant to afford $60 \mathrm{mg}$ (90\% yield) of compound ( \pm )-1 as a white solid after treatment with dichloromethane: $\mathrm{mp} 163-165{ }^{\circ} \mathrm{C}$, IR (neat): $3156,1685,1638 \mathrm{~cm}^{-1} ;{ }^{1} \mathrm{H}$ NMR $\left(\mathrm{CD}_{3} \mathrm{OD}\right) \delta 7.36(\mathrm{~d}, J=1.2 \mathrm{~Hz}, 1$ H), $6.49(\mathrm{~d}, J=4.7 \mathrm{~Hz}, 1 \mathrm{H}), 5.42(\mathrm{~m}, 2 \mathrm{H}), 4.17(\mathrm{~d}, J=12.3 \mathrm{~Hz}, 1 \mathrm{H}), 3.77$ (dd, $J=11.9,5.9 \mathrm{~Hz}, 1 \mathrm{H}), 3.69(\mathrm{~d}, J=12.3 \mathrm{~Hz}, 1$ H), $3.41(\mathrm{dd}, J=11.9,8.8 \mathrm{~Hz}, 1 \mathrm{H}), 1.82(\mathrm{~d}, J=1.2 \mathrm{~Hz}, 3 \mathrm{H}), 1.67(\mathrm{~m}, 1 \mathrm{H}), 1.02(\mathrm{ddd}, J=8.8,5.9,3.9 \mathrm{~Hz}, 1 \mathrm{H}) ;{ }^{13} \mathrm{C} \mathrm{NMR}$ $\left(\mathrm{CD}_{3} \mathrm{OD}\right) \delta 166.6,152.7,145.6,139.6,127.6,111.5,61.7,61.1,44.5,40.7,33.0,12.4 ; \mathrm{FAB} \mathrm{MS} \mathrm{m} / \mathrm{z}$ (relative intensity): 265 $\left(100, \mathrm{MH}^{+}\right)$. Anal. Calcd. for $\mathrm{C}_{13} \mathrm{H}_{16} \mathrm{~N}_{2} \mathrm{O}_{4} \cdot 0.4 \mathrm{H}_{2} \mathrm{O}: \mathrm{C}, 57.43 ; \mathrm{H}, 6.27 ; \mathrm{N}, 10.10$. Found: $\mathrm{C}, 57.63 ; \mathrm{H}, 5.97 ; \mathrm{N}, 9.83$. 


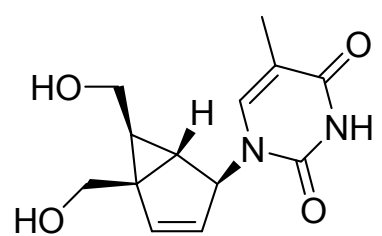

$( \pm)-1$

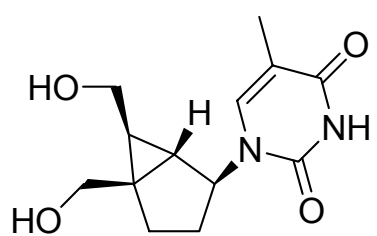

$( \pm)-2$

1-[5,6-Bis(hydroxymethyl)bicyclo[3.1.0]hex-2-yl]-5-methyl-1,3-dihydropyrimidine-2,4-dione (( \pm )-2). A stirred solution of $( \pm)-1(41 \mathrm{mg}, 0.155 \mathrm{mmol})$ in methanol $(10 \mathrm{~mL})$ was reduced under a hydrogen-filled balloon atmosphere in the presence of 5 $\% \mathrm{Pd} / \mathrm{C}(17 \mathrm{mg})$ for $30 \mathrm{~min}$. The reaction mixture was filtered through a Celite ${ }^{\circledR}$ pad and the filtrate concentrated under vacuum. The residue was purified by silica gel flash chromatography $\left(\mathrm{CHCl}_{3}: \mathrm{MeOH}, 24: 1\right)$ to give $( \pm)-2(41 \mathrm{mg}, 100 \%)$ as a white solid after treatment with ethyl ether: $\mathrm{mp} 207-209^{\circ} \mathrm{C}$, IR (neat): $3423,1673,1652 \mathrm{~cm}^{-1} ;{ }^{1} \mathrm{H}$ NMR $\left(\mathrm{CD}_{3} \mathrm{OD}\right) \delta 7.93$ (q, $J=$ $1.2 \mathrm{~Hz}, 1 \mathrm{H}), 4.93(\mathrm{~d}, \mathrm{~J}=6.8 \mathrm{~Hz}, 1 \mathrm{H}), 4.06(\mathrm{~d}, J=12.0 \mathrm{~Hz}, 1 \mathrm{H}), 3.77(\mathrm{dd}, J=11.7,6.6 \mathrm{~Hz}, 1 \mathrm{H}), 3.73(\mathrm{~d}, J=12.0 \mathrm{~Hz}, 1 \mathrm{H})$, $3.50(\mathrm{dd}, J=11.7,8.2 \mathrm{~Hz}, 1 \mathrm{H}), 2.15(\mathrm{~m}, 1 \mathrm{H}), 1.93-1.80(\mathrm{~m}, 2 \mathrm{H}), 1.86(\mathrm{~d}, J=1.2 \mathrm{~Hz}, 3 \mathrm{H}), 1.65$ (m, $1 \mathrm{H}), 1.31(\mathrm{~m}, 1 \mathrm{H}), 1.19$ (ddd, $J=8.2,6.6,3.5 \mathrm{~Hz}, 1 \mathrm{H}) ;{ }^{13} \mathrm{C}$ NMR $\left(\mathrm{CD}_{3} \mathrm{OD}\right) \delta \delta 166.5,153.0,140.2,111.0,62.5,61.8,59.1,38.4,31.9,31.5,29.5$, 27.9, 12.4; FAB MS m/z (relative intensity): $267\left(100, \mathrm{MH}^{+}\right.$), 249 (44). Anal. Calcd. for $\mathrm{C}_{13} \mathrm{H}_{18} \mathrm{~N}_{2} \mathrm{O}_{4} .0 .4 \mathrm{H}_{2} \mathrm{O}$ : C, 57.09; H, 6.93; N, 10.24.Found: C, 57.05; H, 6.83; N; 9.99. 